2011-8

\title{
On the Acceleration of Explicit Finite Difference Methods for Option Pricing
}

\author{
Stephen O'Sullivan \\ Technological University Dublin, stephen.osullivan@tudublin.ie \\ Conall O'Sullivan \\ University College Dublin
}

Follow this and additional works at: https://arrow.tudublin.ie/scschmatart

Part of the Finance and Financial Management Commons, and the Mathematics Commons

\section{Recommended Citation}

O'Sullivan, S., O'Sullivan, C. (2011) On the acceleration of explicit finite difference methods for option pricing, Quantitative Finance 2011 (8), 1177-1191 DOI: 10.1080/14697680903055570

This Article is brought to you for free and open access by the School of Mathematics at ARROW@TU Dublin. It has been accepted for inclusion in Articles by an authorized administrator of ARROW@TU Dublin. For more information, please contact arrow.admin@tudublin.ie, aisling.coyne@tudublin.ie,gerard.connolly@tudublin.ie.

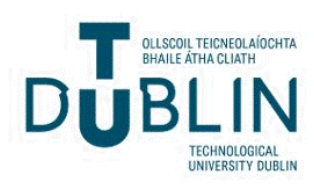


This article was downloaded by: [Dublin City University]

On: 08 November 2011, At: 07:44

Publisher: Routledge

Informa Ltd Registered in England and Wales Registered Number: 1072954 Registered office: Mortimer House, 37-41 Mortimer Street, London W1T 3J H, UK

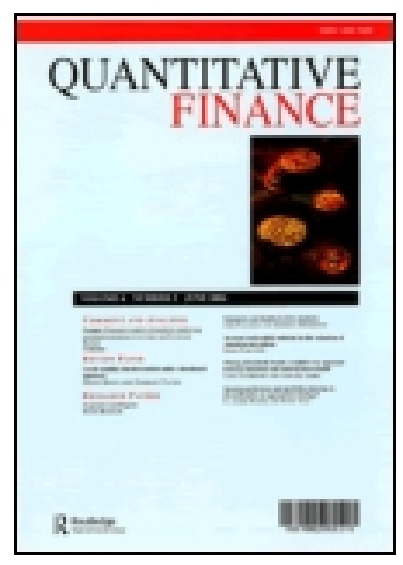

\title{
Quantitative Finance
}

Publication details, including instructions for authors and subscription information: http:// www. tandfonline.com/loi/rquf20

\section{On the acceleration of explicit finite difference methods for option pricing}

\author{
Stephen O'Sullivan ${ }^{a b} \&$ Conall O'Sullivan ${ }^{c}$ \\ ${ }^{a}$ School of Mathematical Sciences, Dublin City University, Glasnevin, Dublin 9, Ireland \\ ${ }^{b}$ UCD School of Mathematical Sciences, University College Dublin, Belfield, Dublin 4, \\ Ireland \\ ' Department of Banking and Finance, The Michael Smurfit School of Business, University \\ College Dublin, Blackrock, Co. Dublin, Ireland
}

Available online: 02 Nov 2009

To cite this article: Stephen O'Sullivan \& Conall O'Sullivan (2011): On the acceleration of explicit finite difference methods for option pricing, Quantitative Finance, 11:8, 1177-1191

To link to this article: http:// dx. doi.org/ 10.1080/ 14697680903055570

\section{PLEASE SCROLL DOWN FOR ARTICLE}

Full terms and conditions of use: http://www.tandfonline.com/page/terms-and-conditions

This article may be used for research, teaching, and private study purposes. Any substantial or systematic reproduction, redistribution, reselling, loan, sub-licensing, systematic supply, or distribution in any form to anyone is expressly forbidden.

The publisher does not give any warranty express or implied or make any representation that the contents will be complete or accurate or up to date. The accuracy of any instructions, formulae, and drug doses should be independently verified with primary sources. The publisher shall not be liable for any loss, actions, claims, proceedings, demand, or costs or damages whatsoever or howsoever caused arising directly or indirectly in connection with or arising out of the use of this material. 


\title{
On the acceleration of explicit finite difference methods for option pricing
}

\author{
STEPHEN O'SULLIVAN*+† and CONALL O'SULLIVAN§ \\ $†$ School of Mathematical Sciences, Dublin City University, Glasnevin, Dublin 9, Ireland \\ FUCD School of Mathematical Sciences, University College Dublin, Belfield, Dublin 4, Ireland \\ $\S$ Department of Banking and Finance, The Michael Smurfit School of Business, \\ University College Dublin, Blackrock, Co. Dublin, Ireland
}

(Received 4 July 2008; in final form 18 May 2009)

\begin{abstract}
Implicit finite difference methods are conventionally preferred over their explicit counterparts for the numerical valuation of options. In large part the reason for this is a severe stability constraint known as the Courant-Friedrichs-Lewy (CFL) condition which limits the latter class's efficiency. Implicit methods, however, are difficult to implement for all but the most simple of pricing models, whereas explicit techniques are easily adapted to complex problems. For the first time in a financial context, we present an acceleration technique, applicable to explicit finite difference schemes describing diffusive processes with symmetric evolution operators, called Super-Time-Stepping. We show that this method can be implemented as part of a more general approach for non-symmetric operators. Formal stability is thereby deduced for the exemplar cases of European and American put options priced under the Black-Scholes equation. Furthermore, we introduce a novel approach to describing the efficiencies of finite difference schemes as semi-empirical power laws relating the minimal real time required to carry out the numerical integration to a solution with a specified accuracy. Tests are described in which the method is shown to significantly ameliorate the severity of the CFL constraint whilst retaining the simplicity of the underlying explicit method. Degrees of acceleration are achieved yielding comparable, or superior, efficiencies to a set of benchmark implicit schemes. We infer that the described method is a powerful tool, the explicit nature of which makes it ideally suited to the treatment of symmetric and non-symmetric diffusion operators describing complex financial instruments including multi-dimensional systems requiring representation on decomposed and/or adaptive meshes.
\end{abstract}

Keywords: Numerical methods for option pricing; Black-Scholes model; Computational finance; Equity options; American options; Exotic options

\section{Introduction}

The Black-Scholes partial differential equation (PDE) (Black and Scholes 1973, Merton 1973) has become a cornerstone of modern derivatives pricing. In general, however, it is rare to find a closed-form solution to the PDE except for the well-known classical cases, such as European call and put options. When a closed-form solution does not exist, for example in the case of American put options, one popular way to proceed is to solve the PDE numerically using finite difference methods (see, for example, Wilmott et al. 1995 and Tavella and

*Corresponding author. Email: steve.osullivan@dcu.ie
Randall 2000). Alternative approaches to American option pricing include binomial tree methods or quasianalytical approximations. The reader is referred to Broadie and Detemple (1996) for a review and comparison of numerical techniques as applied to American put option pricing and Zhu (2006) for a recent semiclosed-form exact solution to the American put option price which consists of an infinite series expansion.

Numerical valuations of options using finite difference methods based on implicit discretizations are usually superior in terms of efficiency to approaches based on conventional explicit discretizations. The principal reason for this is the famous Courant-Friedrichs-Lewy (CFL) stability constraint on explicit schemes which limits the size of the time-step relative to the square of the 
spatial step. In this paper the restriction that the CFL constraint imposes is reduced significantly using an acceleration technique for explicit algorithms, known as Super-Time-Stepping (STS), which is completely novel to computational finance. The technique is applied to the problem of pricing European and American put option prices and compared with a number of standard finite difference methods used frequently in the literature. We demonstrate formal stability by appealing to an extended method developed by the authors which does not have the same dependency on having a highly symmetric evolution operator. Furthermore, we propose that this may be the method of choice for more complex pricing models for which high degrees of symmetry in the evolution operator may not be guaranteed.

It is demonstrated that the efficiencies attained are comparable, and often superior, to those of common implicit differencing techniques. Crucially, this acceleration is achieved without any significant increase in implementation complexity relative to the underlying standard explicit scheme.

The paper is divided into six sections. Section 2 reviews a number of standard finite difference methods used frequently in the literature. Section 3 introduces the Super-Time-Stepping technique whose application to options pricing is the main contribution of this paper. Section 4 describes the novel methodology used to compare the different algorithms. Section 5 contains the results and finally in section 6 we offer concluding remarks.

\section{Review}

This section contains a brief review of the Black-Scholes PDE and the standard finite difference methods, popular in the literature, that are used to solve this PDE for the case of European and American put options.

\subsection{Black-Scholes partial differential equation}

Let $S$ be the asset price underlying the option at time $t$ whose dynamics are described by a geometric Brownian motion. Let $K$ be the exercise price, $T$ the time to expiry, $r$ the risk-free interest rate, and $\sigma$ the volatility of the asset. Denoting the option price as $V(S, t)$, the BlackScholes PDE for European call and put options is then given by

$$
\frac{\partial V}{\partial t}+\frac{1}{2} \sigma^{2} S^{2} \frac{\partial^{2} V}{\partial S^{2}}+r S \frac{\partial V}{\partial S}=r V, \quad 0 \leq t<T,
$$

with the payoff function

$$
V(S, T)= \begin{cases}\max (S-K, 0), & \text { for a call, } \\ \max (K-S, 0), & \text { for a put, }\end{cases}
$$

and the boundary conditions

$$
V(0, t) \approx \begin{cases}0, & \text { for a call } \\ K \mathrm{e}^{-r(T-t)}, & \text { for a put }\end{cases}
$$

$$
V\left(S_{\infty}, t\right) \approx \begin{cases}S, & \text { for a call } \\ 0, & \text { for a put. }\end{cases}
$$

American options can be exercised before expiry and it is never optimal to exercise an American call option before expiry on a stock with no dividend (Merton 1973). However, it may be optimal to exercise an American put option before expiry. The early exercise constraint means that, in the continuation region, the value of an American put option, $V_{A}(S, t)$, satisfies equation (1). However, the exercise region (where it is optimal to exercise early) induces the following payoff and boundary conditions for the American put option on a stock with no dividend:

$$
\begin{aligned}
V(S, T) & =\max (K-S, 0), \quad S \geq 0, \\
\frac{\partial V}{\partial S}(\bar{S}, t) & =-1, \\
V(\bar{S}(t), t) & =K-\bar{S}(t), \\
\lim _{S \rightarrow \infty} V(S, t) & =0, \\
\bar{S}(T) & =K, \\
V(S, t) & =K-S, \quad 0 \leq S<\bar{S}(t),
\end{aligned}
$$

where $\bar{S}(t)$ represents the free and moving early exercise boundary that separates the continuation region from the early exercise region (Wilmott et al. 1995, Duffie 1996).

\subsection{Numerical methods}

Discretizing derivatives in the stock price $S$ in equation (1) via three-point central differencing results in a semidiscrete representation

$$
\frac{\partial V}{\partial t}+\frac{1}{2} \sigma^{2} S_{j}^{2}\left[\frac{\delta_{S}^{2} V_{j}}{\Delta S^{2}}\right]+r S_{j}\left[\frac{\Delta_{S} V_{j}}{2 \Delta S}\right]-r\left[V_{j}\right]=0,
$$

on the domain $\left[0, S_{\infty}\right] \times[0, T]$, where

$$
\begin{aligned}
\delta_{S}^{2} V_{j} & =V_{j+1}-2 V_{j}+V_{j-1}, \\
\Delta_{S} V_{j} & =V_{j+1}-V_{j-1}, \\
S_{j} & =j \Delta S, \quad \text { for } j=\{0,1, \ldots, J\} .
\end{aligned}
$$

All finite difference methods in the present work will be derived from this equation and will therefore converge as $\Delta S^{2}$ to the same exact solution of equation (11). It must be noted that the Black-Scholes PDE is not transformed in any way so the following analysis is as general as possible. Let $\Delta t=T / N$ and $t_{n}=n \Delta t$ for $n=\{0,1, \ldots, N\}$. This notation means $V_{j}^{N}=V\left(S_{j}, T\right)$ is the payoff of the option at maturity and $V_{j}^{0}=V\left(S_{j}, 0\right)$ is the option price at $t=0$ (corresponding to current time). More generally, working backwards recursively through the computational mesh it means that, at time $t_{n+1}$, the values for $V^{n+1}$ are known and the values for $V^{n}$ must be found. Admixing equation (11) at time levels 
$t_{n}$ and $t_{n+1}$ results in the semi-discrete form of the well known $\theta$-method,

$$
\begin{aligned}
\frac{\partial V}{\partial t} & +\frac{1}{2} \sigma^{2} S_{j}^{2}\left[\theta \frac{\delta_{S}^{2} V_{j}^{n+1}}{\Delta S^{2}}+(1-\theta) \frac{\delta_{S}^{2} V_{j}^{n}}{\Delta S^{2}}\right] \\
& +r S_{j}\left[\theta \frac{\Delta_{S} V_{j}^{n+1}}{2 \Delta S}+(1-\theta) \frac{\Delta_{S} V_{j}^{n}}{2 \Delta S}\right] \\
& -r\left[\theta V_{j}^{n+1}+(1-\theta) V_{j}^{n}\right]=0 .
\end{aligned}
$$

This expression may be written compactly as

$$
\frac{\partial \tilde{\mathbf{V}}}{\partial t}-\tilde{\mathbf{P}}\left[\theta \tilde{\mathbf{V}}^{n+1}+(1-\theta) \tilde{\mathbf{V}}^{n}\right]=0,
$$

where $\tilde{\mathbf{P}} \in \mathbb{R}^{J-1} \times \mathbb{R}^{J+1}$ is a tri-diagonal matrix given by

$$
\tilde{\mathbf{P}}=\left[\begin{array}{ccccc}
a_{1} & b_{1} & c_{1} & \cdots & 0 \\
\vdots & \ddots & \ddots & \ddots & \vdots \\
0 & \cdots & a_{J-1} & b_{J-1} & c_{J-1}
\end{array}\right]
$$

with

$$
\begin{aligned}
& a_{j}=\frac{1}{2} r \frac{S_{j}}{\Delta S}-\frac{1}{2} \sigma^{2} \frac{S_{j}^{2}}{\Delta S^{2}}, \\
& b_{j}=r+\sigma^{2} \frac{S_{j}^{2}}{\Delta S^{2}}, \\
& c_{j}=-\frac{1}{2} r \frac{S_{j}}{\Delta S}-\frac{1}{2} \sigma^{2} \frac{S_{j}^{2}}{\Delta S^{2}},
\end{aligned}
$$

and where $\tilde{\mathbf{V}}^{n} \in \mathbb{R}^{J+1}$ is the column vector of option prices at time $t_{n}$ given by

$$
\tilde{\mathbf{V}}^{n}=\left[\begin{array}{c}
V_{0}^{n} \\
\vdots \\
V_{J}^{n}
\end{array}\right] .
$$

Discretizing the temporal derivative $\partial V / \partial t$ to first order as $\left(V_{j}^{n+1}-V_{j}^{n}\right) / \Delta t$ yields the fully discretized form of the $\theta$-method

$$
(\mathbf{I}+\Delta t(1-\theta) \tilde{\mathbf{P}}) \tilde{\mathbf{V}}^{n}=(\mathbf{I}-\Delta t \theta \tilde{\mathbf{P}}) \tilde{\mathbf{V}}^{n+1} .
$$

Applying the boundary conditions at $S_{0}$ and $S_{J}$ means we know the option values $V_{0}^{n}$ and $V_{J}^{n}$ for all $n$. Hence we can recast equations (14) and (20) as

$$
\frac{\partial \mathbf{V}}{\partial t}-\mathbf{P}\left[\theta \mathbf{V}^{n+1}+(1-\theta) \mathbf{V}^{n}\right]-\left[\theta \mathbf{b}^{n+1}+(1-\theta) \mathbf{b}^{n}\right]=0,
$$

and

$$
\begin{gathered}
(\mathbf{I}+\Delta t(1-\theta) \mathbf{P}) \mathbf{V}^{n}+\Delta t(1-\theta) \mathbf{b}^{n} \\
=(\mathbf{I}-\Delta t \theta \mathbf{P}) \mathbf{V}^{n+1}-\Delta t \theta \mathbf{b}^{n+1},
\end{gathered}
$$

respectively, where $\mathbf{P} \in \mathbb{R}^{J-1} \times \mathbb{R}^{J-1}$ is a square nonsymmetric matrix consisting of the $J-1$ innermost columns of $\mathbf{P}$

$$
\mathbf{P}=\left[\begin{array}{cccc}
b_{1} & c_{1} & \cdots & 0 \\
\vdots & \ddots & \ddots & \vdots \\
0 & \cdots & a_{J-1} & b_{J-1}
\end{array}\right],
$$

and where $\mathbf{V}^{n}, \mathbf{b}^{n} \in \mathbb{R}^{J-1}$ are column vectors given by

$$
\mathbf{V}^{n}=\left[\begin{array}{c}
V_{1}^{n} \\
V_{2}^{n} \\
\vdots \\
V_{J-2}^{n} \\
V_{J-1}^{n}
\end{array}\right]
$$

and

$$
\mathbf{b}^{n}=\left[\begin{array}{c}
a_{1} V_{0}^{n} \\
0 \\
\vdots \\
0 \\
c_{J-1} V_{J}^{n}
\end{array}\right] .
$$

When $\theta=1$ this is an explicit scheme that is accurate to $O\left(\Delta t, \Delta S^{2}\right)$. The explicit method is very simple to implement, however the stability of the method depends on the size of the time-step, the spatial step and the coefficients in the PDE (see Wilmott et al. 1995 and Tavella and Randall 2000 for further details on these conditions). In particular, it is required that

$$
\Delta t \leq \frac{\Delta S^{2}}{\sigma^{2} S_{\infty}^{2}},
$$

where $S_{\infty}=S_{J}$ is the maximum price on the computational mesh. This is known as the Courant-FriedrichsLewy (CFL) stability constraint and may be severely restrictive. By way of illustration, if we want to improve accuracy by halving the spatial step we must reduce the time-step by a factor of 4 and computation time goes up by a factor of 8 .

When $\theta=0$ the scheme is fully implicit and is accurate to $O\left(\Delta t, \Delta S^{2}\right)$. The fully implicit scheme has no limitations on the size of the time-step for the method to converge.

When $\theta=\frac{1}{2}$ the resultant scheme is known as the Crank-Nicolson (CN) method and is accurate to $O\left(\Delta t^{2}, \Delta S^{2}\right)$. Similarly to the fully implicit method, CN has no limitations on the size of the time-step for stability. $\mathrm{CN}$ schemes are therefore a frequently favoured method in the literature.

In this paper we shall additionally employ Richardson Extrapolation (RE) to render explicit and the fully implicit schemes second-order accurate in time. RE is carried out on a step-wise basis as follows.

We assume a smoothly convergent first-order accurate method for the temporal integration of the semi-discrete equation (21) with exact solution $V_{\Delta S}(S, t)$. Given a second-order accurate solution at time level $n+1$ such that $\quad V_{j}^{n+1}=V_{\Delta S}(j \Delta S,(n+1) \Delta t)+(N-n-1) \mathcal{O}\left(\Delta t^{3}\right)$ we may take a single step of size $\Delta t$ to approximate the solution at time level $n$ using $V_{j}^{n}(\Delta t)=V_{\Delta S}(j \Delta S, n \Delta t)+$ $C \Delta t^{2}+\mathcal{O}\left(\Delta t^{3}\right)$ for some constant $C$. Similarly, taking two steps of size $\Delta t / 2$, we have $V_{j}^{n}(\Delta t / 2)=$ $V_{\Delta S}(j \Delta S, n \Delta t)+(C / 2) \Delta t^{2}+\mathcal{O}\left(\Delta t^{3}\right)$. Subtracting the expression for $V_{j}^{n}(\Delta t)$ from twice the expression for 
$V_{j}^{n}(\Delta t / 2)$ yields a second-order advancement in the solution from time level $n+1$ to level $n$ according to

$$
V_{j}^{n}=2 V_{j}^{n}(\Delta t / 2)-V_{j}^{n}(\Delta t) .
$$

This is the prescription of the RE we employ as opposed to the more usual post-processed form which requires two independently derived solutions for use in the extrapolation $V_{j}^{0}=2 V_{j}^{0}(\Delta t / 2)-V_{j}^{0}(\Delta t)$ (e.g., Geske and Johnson 1984). The significant difference is that, in the former case, a properly second-order integration method is obtained in the sense that a second-order solution is available at all intermediate times.

The use of RE comes at the expense of an increase in the computational workload. However, it is simple to implement and of greater applicability than $\mathrm{CN}$, as we shall see.

For implicit schemes, the system of simultaneous equations (22) may be solved exactly via direct matrix inversion. For the vanilla option pricing problems under consideration in this work, the Brennan and Schwartz (1977) algorithm may be reformulated to employ LU-decomposition (Ikonen and Toivanen 2007a). While this is an $\mathcal{O}(N)$ method, it does not generalize well. For example, in the relatively simple case of pricing American put options under Heston's stochastic volatility model, the early exercise region must take a specific form for the Brennan and Schwartz algorithm to work. Since we are interested in comparison of methods of general applicability and examine one-dimensional vanilla options for the purposes of bench-testing computational efficiency only, we do not consider the Brennan and Schwartz class of methods any further.

Iterative approaches may also be taken to obtain solutions to within some prescribed accuracy. The most popular of these is Successive Over-Relaxation (SOR); see Crank (1984) for more detail on SOR. In the case of American options, the early exercise constraint requires the use of a variation known as Projected SOR (PSOR) (Wilmott et al. 1995, Ikonen and Toivanen 2007b). Other iterative methods such as the Gauss-Seidel, originally used by Brennan and Schwartz (1977) in the context of finite difference methods applied to American options pricing, and the Jacobi method are also discussed by Wilmott et al. (1995). Using iterative methods can speed up implicit finite difference schemes relative to direct matrix inversion, particularly when fast inversion techniques such as LU-decomposition are unavailable.

When pricing American put options we have to consider the possibility of early exercise. In explicit schemes this is easily handled recursively as follows. Assume the American constraint has been applied at timestep $t_{n+1}$. The unknown value $V_{j}^{n}$ is calculated from the known values $V_{j-1}^{n+1}, V_{j}^{n+1}$ and $V_{j+1}^{n+1}$ and then we replace $V_{j}^{n}$ with $\max \left(V_{j}^{n}, K-S_{j}\right)$.

On the other hand, when solving implicitly for the unknowns $V_{j}^{n}$, we must take into account that the value at any point $j$ may be equal to the corresponding continuation values if early exercise is sub-optimal, or the early exercise value if early exercise is optimal.
Therefore, it is clearly inappropriate to first find the continuation value $V_{j}^{n}$ and replace it with $\max \left(V_{j}^{n}, K-S_{j}\right)$ for all $j$ since all of these values are coupled and any such replacement must be made simultaneously for all $j$. Because of this, direct matrix inversion methods can be at best first-order accurate in time and iterative algorithms, such as PSOR, are necessary. During each PSOR iteration, the projection is carried out by replacing $V_{j}^{n}$ with $\max \left(V_{j}^{n}, K-S_{j}\right)$. Over several iterations this converges to simultaneous early exercise enabling convergence at rates above first order in time.

In the next section we introduce an accelerated explicit finite difference scheme, known as Super-Time-Stepping, that is completely novel to computational finance. This scheme reduces significantly the restriction that the CFL constraint imposes on the size of the time-step relative to the spatial step in conventional explicit finite difference schemes. We go on to describe how STS may be implemented as a component of a composite method with stability properties appropriate for more general problems. We invoke RE for second-order accuracy in time. In the subsequent section the accelerated explicit scheme is applied to European and American put options and is demonstrated to be of comparable or superior efficiency to a number of implicit differencing schemes with no significant increase in implementation complexity relative to standard explicit schemes.

\section{Acceleration methods}

\subsection{Super-Time-Stepping}

Super-Time-Stepping (STS) is a technique that can be used to accelerate explicit schemes for parabolic problems. In the following, we shall use the description of Alexiades et al. (1996), itself a variant of a method presented by Gentzsch (1979) and essentially a pareddown Runge-Kutta-Chebyshev (RKC) method (van der Houwen 1977, van der Houwen and Sommeijer 1980, Verwer et al. 1990, Verwer 1996, Sommeijer et al. 1997).

Despite the fact that the STS method is approximately 30 years old, it is extraordinary that it has been reported in use by few researchers. The very limited number of numerical investigations we are aware of employing STS are in engineering and physical disciplines and include: nonlinear degenerate convection-diffusion (Evje et al. 2001); electromagnetic wave scattering (Shi et al. 2006); isotropic and anisotropic diffusion on biological membranes (Sbalzarini et al. 2006); and magnetic field diffusion in astrophysics (Mignone et al. 2007, O'Sullivan and Downes 2007). To our knowledge, it has received no attention to date in the area of finance.

The essence of STS is that rather than requiring stability at each step of the integration, $N_{\text {STS }}$ sub-steps of varying size $\Delta t_{j}$ are rolled together into a single superstep $\Delta t_{\text {STS }}$ according to

$$
\Delta t_{\mathrm{STS}}=\sum_{j=1}^{N_{\mathrm{STS}}} \Delta t_{j},
$$


and stability is only demanded at the end of the superstep. $\dagger$ To proceed, we assume a linear scheme on $\mathbf{V} \in \mathbb{R}^{M}$ of the form

$$
\mathbf{V}^{n}=\left(\mathbf{I}-\Delta t_{\mathrm{STS}} \mathbf{A}\right) \mathbf{V}^{n+1}=\left[\prod_{j=1}^{N_{\mathrm{STS}}}\left(\mathbf{I}-\Delta t_{j} \mathbf{A}\right)\right] \mathbf{V}^{n+1},
$$

where the solutions at time levels $n+1$ and $n$ are known and unknown, respectively, $\mathbf{I}$ is the identity matrix and $\mathbf{A} \in \mathbb{R}^{M} \times \mathbb{R}^{M}$ is a symmetric positive definite matrix. It is well known that, for stability, we must have

$$
\left|\prod_{j=1}^{N_{\mathrm{STS}}}\left(\mathbf{I}-\Delta t_{j} \lambda\right)\right|<1,
$$

for all eigenvectors $\lambda$ of $\mathbf{A}$.

The properties of Chebyshev polynomials of degree $N_{\text {STS }}$ (Markoff 1916) then allow us to explicitly enforce stability while maximizing $\Delta t_{\mathrm{STS}}$ to provide a set of optimal values for the sub-steps given by

$$
\Delta t_{j}=\Delta t_{\mathrm{STD}}\left[(-1+v) \cos \left(\frac{2 j-1}{N_{\mathrm{STS}}} \frac{\pi}{2}\right)+1+v\right]^{-1},
$$

where $\Delta t_{\mathrm{STD}}$ is the normal explicit time-step limit and $v$ is a damping factor. Note, in particular, that

$$
\Delta t_{\mathrm{STS}} \rightarrow N_{\mathrm{STS}}^{2} \Delta t_{\mathrm{STD}}, \quad \text { as } v \rightarrow 0 .
$$

While this scheme is stable for any choice of $N_{\mathrm{STS}}$ given a large enough value of $v$, in practice a balance can be struck between the two parameters to optimize the performance of the scheme. The method is unstable in the limit $v=0$. We illustrate the efficacy of the acceleration process for $N_{\mathrm{STS}}=30$ in figure 1 . It can be seen that the first substep may be up to 25 times the stable limit for a standard explicit integration as $v \rightarrow 0$, but subsequent substeps become increasingly small. The effect of this is a cumulative error cancellation that recovers stability over the composite superstep. Crucially, there is a net payoff in terms of the size of the superstep with respect to $N_{\mathrm{STS}}$ steps of size $\Delta t_{\mathrm{STD}}$ as described by equation (32).

Note that although formal results only exist for linear schemes, there is ample evidence, as described above, that nonlinear target systems are equally amenable to the STS method.

It can be shown (Alexiades et al. 1996) that STS is essentially first order in time. It is not possible to introduce additional temporal structure to an STS step since intermediate values obtained during a STS cycle are physically meaningless and may not be used as approximations to the solution in any sense. Therefore, predictor-corrector-style methods are not applicable should higher-order convergence be required. On the other hand, we have found that RE works perfectly well. By this method all the advantages of the first-order STS method are easily transferred to second- (or higher-) order schemes.

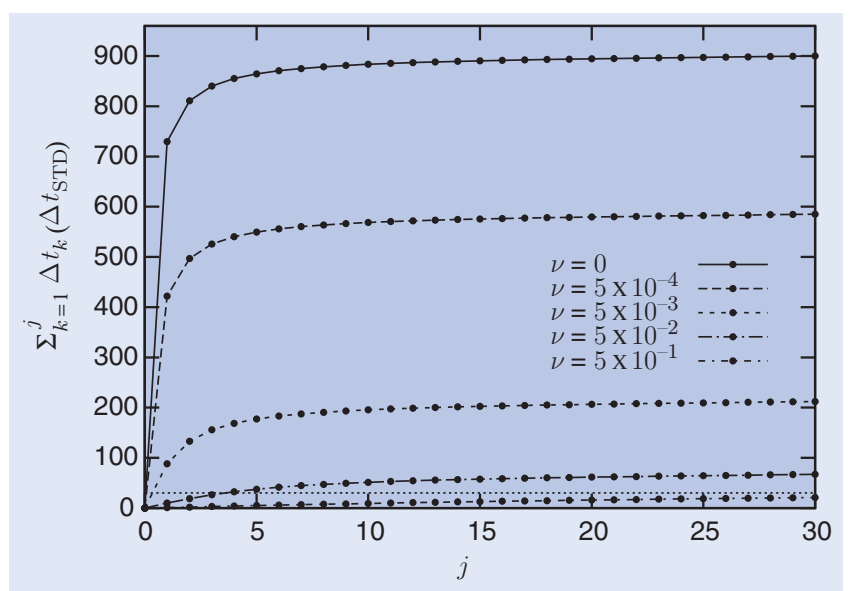

Figure 1. Illustration of acceleration via STS. Accumulated time $\sum_{k=1}^{j} \Delta t_{k}$ is shown in units of standard explicit time-step $\Delta t_{\mathrm{STD}}$ over single superstep $\Delta t_{\mathrm{STS}}$ with $N_{\mathrm{STS}}=30$ for a range of damping factors $v$. A reference line at $N_{\mathrm{STS}} \Delta t_{\mathrm{STD}}$ indicates the time attained over $N_{\text {STS }}$ unaccelerated (standard explicit) steps. Note that acceleration approaches $N_{\mathrm{STS}}$ times this value as $v \rightarrow 0$, in agreement with equation (32). Note also that deceleration occurs for the highest considered damping factor of $v=0.5$. In this work, $v=5 \times 10^{-4}$ and $N_{\mathrm{STS}}=30$ for all tests.

The principal advantage of the STS method is not efficiency, however, but simplicity. Explicit discretizations of even the most complex systems of parabolic equations are very straightforward. In particular, implementation of adaptive mesh refinement (AMR) technologies and/or parallelization via domain decomposition techniques present no great challenges from within an explicit framework. On the contrary, when implicit methods are involved, tackling problems of even a moderate level of complexity can be an exceedingly intricate task.

In the next section the performance of the STS method applied via equation (22) is compared with finite difference schemes described in section 2 .

\subsection{Composite}

Since stability is assured by the above analysis for symmetric positive definite $\mathbf{A}$ in equation (29), we provide a formal stability analysis for an alternative discretization of equation (1) in appendix A. The scheme presented therein is formally stable under application of STS to a split symmetric positive definite operator. In practice, we find that, as suggested by other authors previously (e.g., Alexiades et al. 1996 and O'Sullivan and Downes 2007), this alternative scheme is not strictly necessary when the evolution operator has a dominant symmetric component. For the cases under consideration here, in fact, we find it has negligible impact and therefore it is not used.

We wish to emphasize that, while the composite scheme is employed in this work for the purposes of stability analysis, its greater generality may prove it to be the appropriate choice for the numerical integration of

$\dagger$ It has been claimed by Verwer (1996) that factorized RKC methods are impractical as they suffer from severe internal instability. We find no evidence of this for $N_{\mathrm{STS}} \lesssim 30$. 
systems of equations for which the evolution operator does not display the high degree of symmetry observed for pricing vanilla options under Black-Scholes.

\section{Methodology}

In terms of the real-world usefulness of a numerical scheme $X$ for pricing financial instruments, it is important to recognize that the wall-time (the real time taken to carry out the numerical integration) $W_{X}$ required for a scheme to perform a calculation will depend on the maximum permitted error $E$ of the solution. Since the greatest error between the numerical solution $V_{j}$ and the exact solution $V\left(S_{j}\right)$ arises when $S_{j}$ is the equal to the exercise price $K$, where there is a discontinuity in the first derivative of the payoff function, we use the definition $E \equiv\left|V_{k}^{0}-V(K, 0)\right|$ for the error in the numerical solution (where the index $k$ is the index corresponding to the spot price $S_{k}=K$ ). For consistency with this measure, the SOR schemes are required to converge to within a userprescribed tolerance on $V_{k}^{n}$ rather than over a range of values as is more usual. From this point on, we shall generally omit the $X$ subscript from quantities associated with a scheme $X$ for clarity of notation. Dependent variables will be implicitly assumed to be associated with a given scheme.

The schemes that are used as benchmarks for comparison with the STS explicit scheme with Richardson extrapolation (STS_RE) are as follows: the standard explicit scheme with RE (STD_RE), the fully implicit scheme that uses matrix inversion and Richardson extrapolation (INV_RE), the fully implicit scheme that uses SOR and RE (SOR_RE), the CN scheme that uses matrix inversion (INV_CN) and the $\mathrm{CN}$ scheme that uses SOR (SOR_CN). Scheme comparison is traditionally performed by presenting timings for solutions obtained to non-uniform accuracies, although Broadie and Detemple (1996) go further by considering simultaneously timings and accuracy of the pricing methods they considered. Presenting timings for solutions obtained to non-uniform accuracies is not a fair comparison of the efficiencies as clearly a scheme which has run to a higher accuracy has expended greater computational resources than necessary to achieve a lower value. The efficiency of finite difference schemes which are derived from a single semi-discrete representation (in this case equation (11), the semidiscrete representation of equation (1)) may be represented by three parameters for a given problem, with an additional three parameters necessary to impose the CFL condition on explicit schemes. We now proceed to elucidate this idea by deriving semi-empirical functional forms for the wall-time $W$ as a function of the error $E$.

All schemes under consideration here are second order accurate in price by construction from equation (11). Assuming order $p$ in time the total error is given by

$$
E=E_{t}+E_{S}
$$

where we say

$$
E_{t}=O\left(\Delta t^{p}\right) \approx \zeta \Delta t^{p}, \quad E_{S}=O\left(\Delta S^{2}\right) \approx \eta \Delta S^{2}
$$

are the errors arising from the temporal and price discretization, respectively. Assuming $W$ scales inversely with $\Delta t$ we have

$$
W \propto E_{t}^{-1 / p}
$$

and hence

$$
W=\beta \Delta t^{-1},
$$

for some $\beta$. In fact, we know $p=2$ in all cases bar INV_CN applied to the American put option where $p=1$. Furthermore, if the scaling of the wall-time with $\Delta S$ is given by the power law

$$
W \propto \Delta S^{-2 \psi},
$$

we have

$$
W \propto E_{S}^{-\psi}
$$

As we shall see later, for the schemes considered in this paper, implicit schemes have $\psi \gtrsim 1.1$ and explicit schemes have $\psi \lesssim 0.4$. Note that since all schemes are approximating the solution to the same semi-discrete equation (11), $\eta$ depends only on the problem and not the scheme.

We can write

$$
W=\alpha E_{t}^{-1 / p} E_{S}^{-\psi}
$$

where the parameters $(\alpha, \psi)$ are fit by experiment for a given test case as follows. To determine $\psi$ for each scheme, we run a series of tests for a fixed value of $\Delta t$ at different price resolutions $\Delta S$. Once $\psi$ is known, a series of tests is run for a fixed value of $\Delta S$ at different time resolutions $\Delta t$. The exact solution to equation (11), $V_{\Delta S}$, is estimated via an independent second-order explicit code (Ødegaarde 2007) run to very high accuracy. This code was minimally modified by the authors to attain second-order accuracy in time via RE. The value obtained is then used to estimate $E_{t}=\left|V_{k}(K, 0)-V_{\Delta S}(K, 0)\right|$ from the experimental data which may then be fit to

$$
W=\beta \zeta^{1 / p} E_{t}^{-1 / p}
$$

From equation (39),

$$
\beta=\alpha \zeta^{-1 / p} E_{S}^{-\psi}
$$

and hence $\alpha$ follows directly given $E_{S}=\mid V(K, 0)-$ $V_{\Delta S}(K, 0) \mid$. The exact solution to equation $(1), V(K, 0)$, is derived to high accuracy using an analytical model for European options or, for American options, an independent binomial tree code (Ødegaarde 2007) run to extremely high accuracy.

Minimizing the wall-time $W$ for a fixed total error $E$ determines the dependencies of $E_{t}$ and $E_{S}$ on $E$ according to the relations

$$
E_{t}=\frac{1}{1+\psi p} E, \quad E_{S}=\frac{\psi p}{1+\psi p} E .
$$


Equation (39) may then be recast in the form

$$
W=\alpha \frac{(1+\psi p)^{\psi+1 / p}}{(\psi p)^{\psi}} E^{-\psi-1 / p} .
$$

From this efficiency equation, an approximation to the wall-time required for a scheme $X$ to evaluate the solution for a given problem to a maximum specified accuracy $E$ is characterized by three parameters $(\alpha, \psi, p)$. In a subsequent section we shall derive these parameters for a set of schemes applied to given option pricing problems. Firstly, however, it is necessary to consider an additional constraint on the viable choices for the pair $\left(E_{t}, E_{S}\right)$ in optimizing the efficiency of explicit schemes.

\subsection{Stability constraints on explicit schemes}

For STD_RE, the CFL condition (equation (26)) requires $\Delta t \leq \Delta S^{\overline{2}} /\left(\sigma^{2} S_{\infty}^{2}\right)$ where $S_{\infty}$ is the maximum price on the computational mesh. For a given number of uniformly spaced price mesh points $J$, the maximum stable time-step is $1 /\left(\sigma^{2} J^{2}\right)$. Therefore, in an integration over a time $T$, a minimum number of time-steps $T \sigma^{2} J^{2}$ is required. More generally, for an explicit scheme $X$ this constraint will determine the scheme efficiency for $E \leq E_{\text {crit }}$. The critical point is the lower bound of the regime described by equations (42) where the CFL condition is marginally satisfied, i.e.

$$
\Delta t=\epsilon \Delta S^{2}
$$

Using equations (34) we may then write

$$
E_{\mathrm{crit}}=\left[\frac{1}{\zeta}\left(\frac{\eta}{\epsilon}\right)^{p}\right]^{1 /(p-1)} \frac{1+\psi p}{(\psi p)^{p /(p-1)}}
$$

Note that if $p=1$ then as long as $\eta / 4 \zeta \leq \epsilon$, equations (42) trivially satisfy the CFL condition for all $E$.

We have theoretical values for $\epsilon$ given by

$$
\epsilon_{\text {STD_RE }}^{\text {theory }}=1 / \sigma^{2} S_{\infty}^{2}
$$

and

$$
\epsilon_{\mathrm{STS} \_ \text {RE }}^{\text {theory }}=\frac{N_{\mathrm{STS}}}{2 \sqrt{v}}\left(\frac{(1+\sqrt{v})^{2 N_{\mathrm{STS}}}-(1-\sqrt{v})^{2 N_{\mathrm{STS}}}}{(1+\sqrt{v})^{2 N_{\mathrm{STS}}}+(1-\sqrt{v})^{2 N_{\mathrm{STS}}}}\right) \epsilon_{\mathrm{STD} \_ \text {RE }} .
$$

We shall see later that, in practice, $\epsilon_{\mathrm{STD} \_\mathrm{RE}} \approx \epsilon_{\mathrm{STD} \text {-RE }}^{\text {theory }}$ but $\epsilon_{\text {STS_RE }}>\epsilon_{\text {STS_RE }}^{\text {theory }}$. Thus the theoretical value is conservative in the case of STS RE.

For $E<E_{\text {crit }}$, the relationship between $E_{t}$ and $E_{S}$ is no longer freely tunable for optimal efficiency but set by the marginal CFL condition $\Delta t=\epsilon \Delta S^{2}$ giving $\left(E_{t} / \zeta\right)^{1 / p}=$ $\epsilon E_{S} / \eta$. For $p=2, E=E_{t}+E_{S}$ may be written as a quadratic equation in $E_{S}$ with one admissible root,

$$
\frac{\zeta \epsilon^{p}}{\eta^{p}} E_{S}^{p}+E_{S}-E=0
$$

Equation (39) may then be written as a function of $E_{S}$,

$$
W=\frac{\alpha \eta}{\zeta^{1 / p} \epsilon} E_{S}^{-1-\psi} .
$$

\section{Efficiency tests}

In the following we shall consider European and American put options with $T=1, \sigma=0.2, r=0.05$ and $K=100$. A uniform mesh is assumed in $S$ over the range $[0,5 K]$. The authors have confirmed similar results for other choices of $T, \sigma$, and $r$ and for a uniform mesh in $\log (S)$. All tests are carried out using MATLAB R2007a on a $2.2 \mathrm{GHz}$ Intel Core 2 Duo processor under Fedora 8 linux.

In all tests, SOR_CN and SOR_RE are tuned with an over-relaxation parameter $w=1.1$ and tolerance set to $10^{-12}$. No maximum iteration count is assigned so that this tolerance is always achieved. For STS RE, in all tests $v=5 \times 10^{-4}$ and $N_{\text {STS }}=30$. These values formally imply a limiting stable time-step 380.2961 times longer than that for STD_RE. Convergence to the solution of equation (1) is measured against an analytic solution for European put options and, for American put options, against a high accuracy solution $\left(E \approx 10^{-6}\right)$ obtained from a binomial tree method. To measure convergence rates to the exact solution of equation (11) for a given $\Delta S$, very high accuracy solutions from an independent second-order explicit scheme (Ødegaarde 2007) are used $\left(\Delta t=T / 10^{7}\right)$.

\subsection{Convergence studies: determining $\zeta, \eta, p$}

Convergence studies of all schemes for European and American put option pricing are presented in figures 2 and 3 , respectively.

The scaling of $E_{S}$ is obtained for each scheme for fixed $\Delta t$ and $\Delta S=\left\{5 K /\left(100 \times 2^{n}\right) \mid n=0,1,2,3,4,5,6\right\}$. Given a sufficiently small $\Delta t$, the temporal error $E_{t}$ will be negligible compared with the price error $E_{S}$ and $E=\left|V(K, 0)-V_{j}(K, 0)\right| \approx E_{S}$. We find $\Delta t=T / 1000$ for the implicit solvers fulfills this condition. Stability over the full range of spatial resolutions demands lower values for the explicit schemes: $\Delta t=T / 3600$ for STS_RE and $\Delta t=T / 1660000$ for STD_RE. In the upper panel of figure 2, second-order convergence with $\Delta S$ is confirmed for all schemes pricing the European option. Note that, at the very lowest values of $\Delta S$, the temporal error begins to dominate. While second-order convergence is also clear from the upper panel of figure 3 , the early exercise constraint results in temporal errors that dominate the spatial errors at larger values of $\Delta S$ than in the European case. In particular, the temporal error for INV_CN is dominant at even moderate values of $\Delta S$ because the early exercise constraint reduces this scheme to first-order accuracy in time when an iterative solver is not used (Wilmott et al. 1995).

The temporal order of convergence is determined for a fixed price spacing $\Delta S=5 K / 100$ and a range of temporal resolutions $\Delta t=\left\{T /\left(20 \times 2^{n}\right) \mid n=0,1, \ldots, 12,13\right\}$. In the case of STD_RE, the stable temporal resolutions are restricted and we consider $\Delta t=\left\{T /\left(20 \times 2^{n}\right) \mid\right.$ $n=5,6, \ldots, 14,15\}$. The temporal error $E_{T}$ is then determined from $V_{\Delta S=5 K / 100}$.

The lower panel of figure 2 illustrates the case of the European option pricing test case. All schemes show 


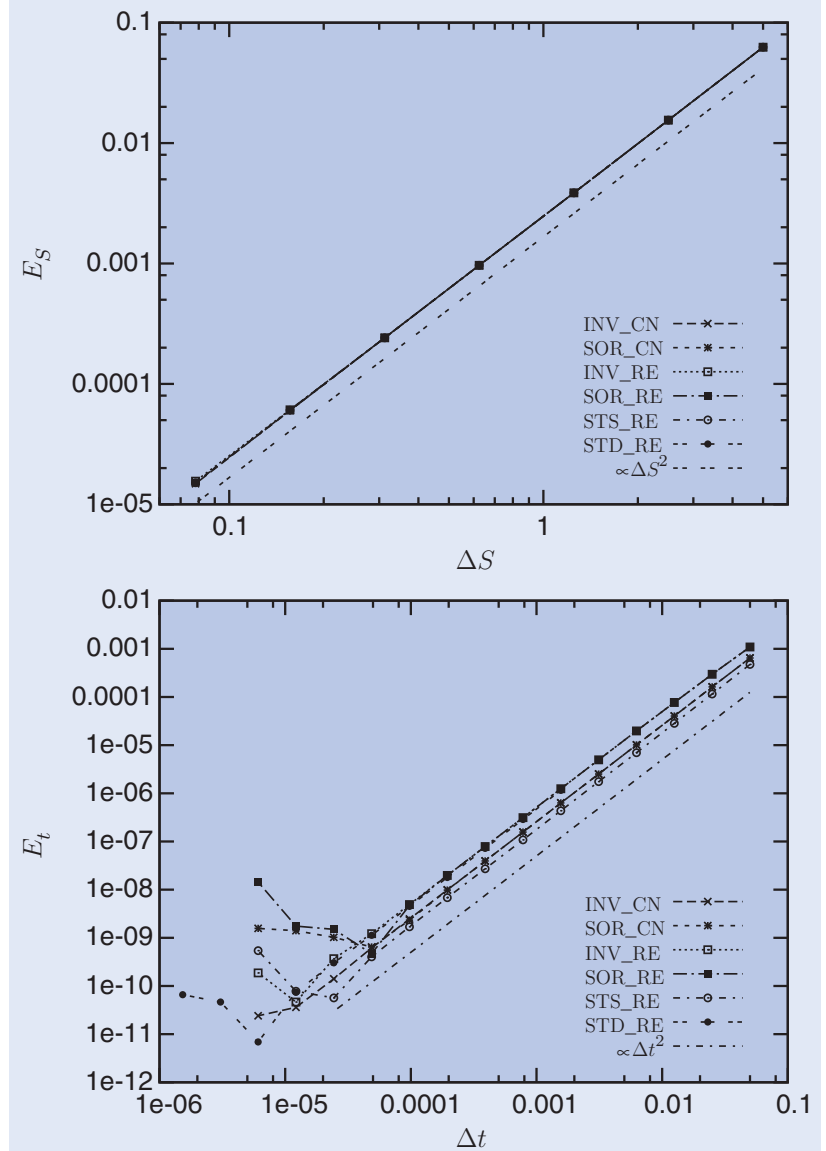

Figure 2. Scheme convergence tests for European put option pricing. Upper panel: Spatial error at the strike price, $E_{S}$, plotted as a function of $\Delta S$. For this test, errors are measured against a value obtained from an exact solution to the Black-Scholes equation. The influence of temporal errors in the numerical integration is minimized by using a sufficiently small value for $\Delta t$ in all cases. Points are almost exactly coincident in all cases. Clearly, all tested schemes converge to the exact solution at second order according to $E_{s}=\eta \Delta S^{2}$ for some $\eta$. Values for $\eta$ are obtained from this data as necessary. A reference line scaling with $\Delta S^{2}$ is shown to guide the eye. Lower panel: Temporal error at the strike price, $E_{t}$, plotted as a function of $\Delta t$ for $\Delta S=5 K / 100$. Temporal errors are calculated using a highaccuracy approximation to the exact solution of equation (11) obtained from an independent code modified to be second order in time (Ødegaarde 2007). It is clear that all schemes converge to this value with second-order accuracy according to $E_{t}=\zeta \Delta t^{2}$, for some $\zeta$, down to levels where machine accuracy becomes important. Values for $\zeta$ are obtained from this data as required. A reference line scaling with $\Delta t^{2}$ is also shown to guide the eye.

second-order convergence with time down to levels where round-off error becomes significant. The results are qualitatively similar for the American case as shown in the lower panel of figure 3 except that, again, the early exercise constraint degrades the smoothness of convergence in all cases except INV_CN, which, as previously commented on, is reduced to first-order accuracy in time. We find good experimental agreement for $p=2$ in all cases except INV_CN applied to the American option problem where $p=1$, as expected.

The power laws given by equations (34) are fit to the well-behaved data-points; as previously observed, other sources of error become significant at the lowest values of

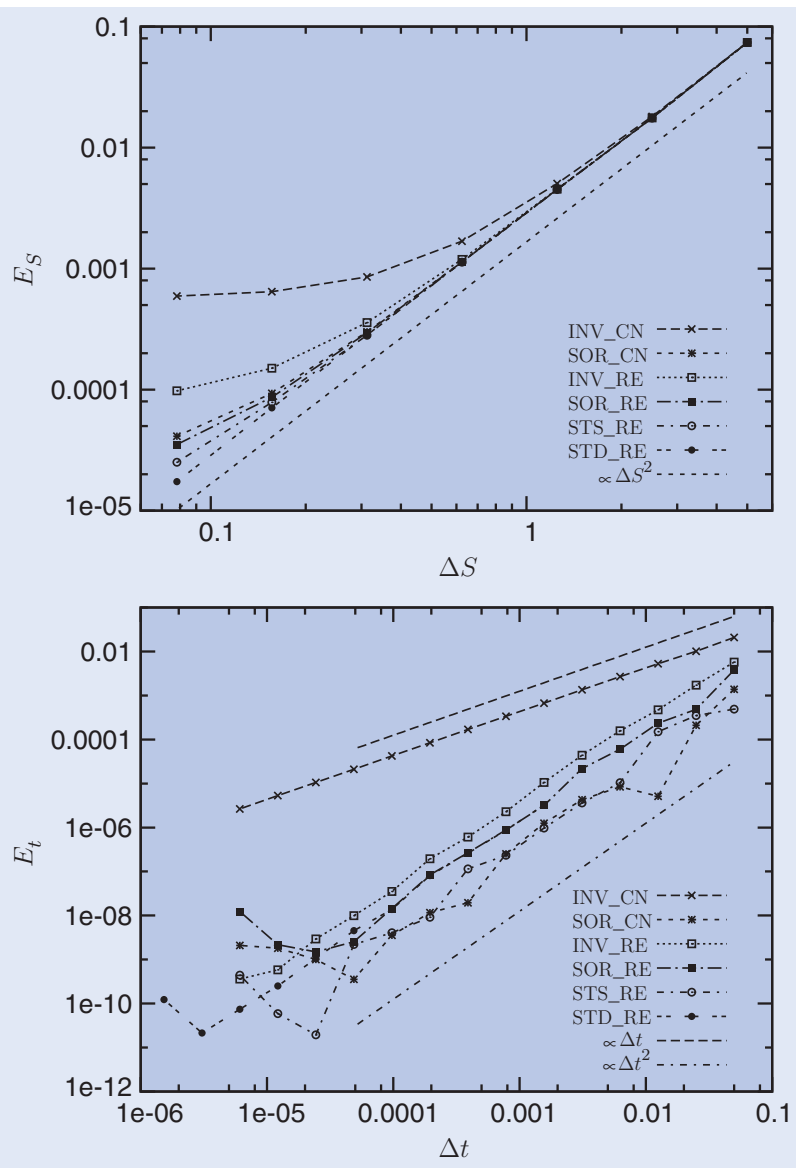

Figure 3. Scheme convergence tests for American put option pricing. Upper panel: Spatial error at the strike price, $E_{S}$, plotted as a function of $\Delta S$. For this test, errors are measured against a high-accuracy approximation to the exact solution using a binomial tree method. The influence of temporal errors in the numerical integration is minimized by using a sufficiently small value for $\Delta t$ in all cases. Down to small $\Delta S$ all schemes, except INV_CN, which is first order in time, demonstrate convergence to the estimated exact solution at second order according to $E_{s}=\eta \Delta S^{2}$ for some $\eta$. Values for $\eta$ are obtained from this data as necessary. A reference line scaling with $\Delta S^{2}$ is shown to guide the eye. Lower panel: Temporal error at the strike price, $E_{t}$, plotted as a function of $\Delta t$ for $\Delta S=5 K / 100$. Temporal errors are calculated using a high-accuracy approximation to the exact solution of equation (11) obtained from an independent code modified to be second order in time (Ødegaarde 2007). Except for INV_CN, which shows first-order temporal accuracy, all schemes converge to this value with approximate secondorder accuracy according to $E_{t}=\zeta \Delta t^{2}$, for some $\zeta$. Values for $\zeta$ are obtained from this data as required. Reference lines scaling with $\Delta t$ and $\Delta t^{2}$ are also shown to guide the eye.

$\Delta t$ and $\Delta S$. The best-fit values obtained for $\zeta$ and $\eta$ are used in the following sections to evaluate the dependent parameters as required. The values corresponding to the explicit schemes are detailed in table 1 as they are required to apply the CFL constraint. This is discussed later in section 5.4 .

\subsection{Wall-time scaling with price resolution: determining $\psi$}

In this section, the scaling of the wall-time required to achieve a given accuracy as a function of the spot price 

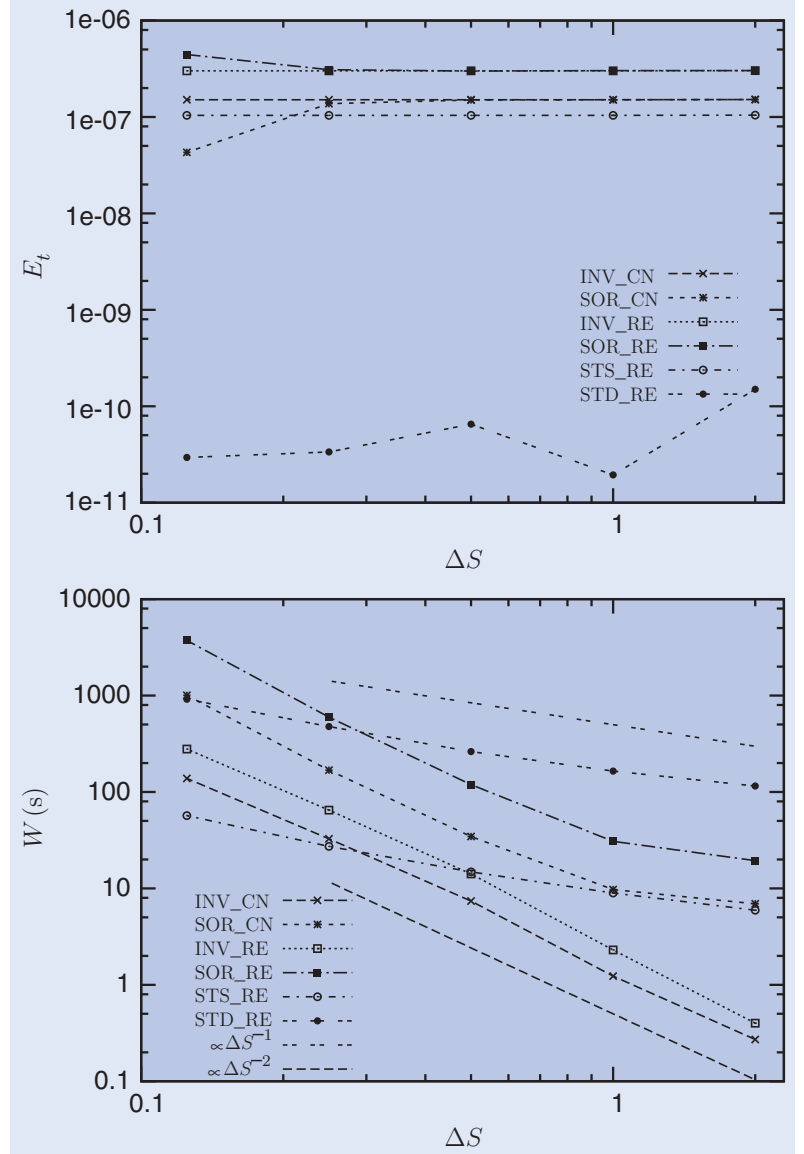

Figure 4. Temporal error and wall-time scaling with price resolution for European put option pricing. Upper panel: Temporal error at the strike price, $E_{t}$, plotted as a function of $\Delta S$ for a fixed value of $\Delta t=T / 1280$ (except for $\Delta t=T / 640000$ in the case of STD_RE). Errors are calculated using a highaccuracy approximation to the exact solution of equation (11) obtained from an independent code modified to be second order in time (Ødegaarde 2007). To a fair approximation, the temporal error has no dependence on the spatial resolution. Lower panel: The wall-time $W$ in seconds as a function of price resolution $\Delta S$ for the tests described above. This data is used to fit the scaling law $W \propto \Delta S^{-2 \psi}$. At moderate to high resolution the law is clearly well obeyed. Reference lines scaling with $\Delta S^{-1}$ and $\Delta S^{-2}$ are also shown.

resolution is assessed. The range of values used for the price mesh spacing is $\Delta S=\left\{5 K /\left(250 \times 2^{n}\right) \mid n=0,1,2\right.$, $3,4\}$. For all schemes, $\Delta t=T / 1280$, except for STD_RE where $\Delta t=T / 640000$ is required for stability. Error and wall-time data for European and American option pricing are presented in figures 4 and 5, respectively. The upper panel in each case shows the dependence of the temporal error $E_{t}$ on $\Delta S$ and the lower panels illustrate the walltime $W$ scaling. Notably, the SOR schemes tend to diverge from a simple power law fit at high values of $\Delta S$, and at low values for $E_{t}$ in the European case. The temporal error $E_{t}$ is particularly erratic for STS_RE applied to the American option. This does not reflect any inherent difficulty with the scheme, but merely indicates that the power law approximation is not well suited to STS_RE with the early exercise constraint applied in this way. We defer discussion of the scheme's performance under the more modern penalty method approach to
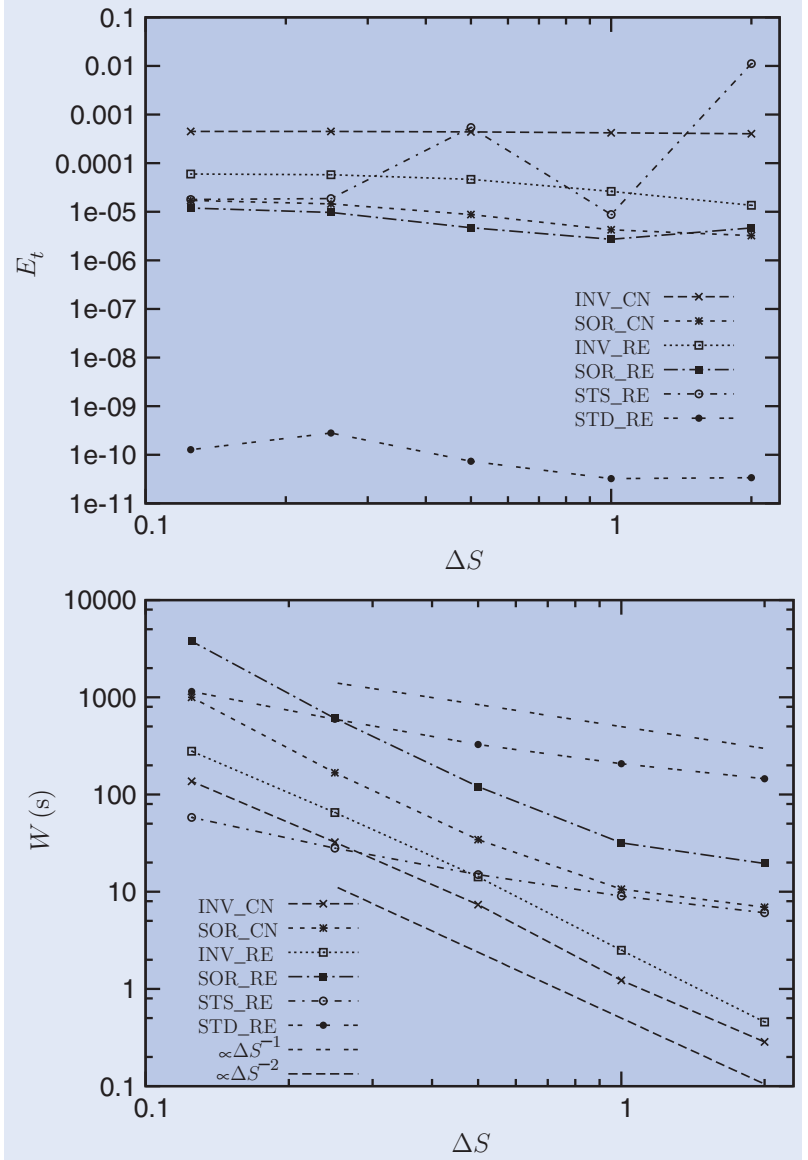

Figure 5. Temporal error and wall-time scaling with price resolution for American put option pricing. Upper panel: Temporal error at the strike price, $E_{t}$, plotted as a function of $\Delta S$ for a fixed value of $\Delta t=T / 1280$ (except for $\Delta t=T / 640000$ in the case of STD_RE). Errors are calculated using a highaccuracy approximation to the exact solution of equation (11) obtained from an independent code modified to be second order in time (Ødegaarde 2007). Except for STS_RE at low to moderate values of $\Delta S$, which shows substantial volatility in $E_{t}$ due to the implementation of the early exercise constraint, the temporal error is reasonably approximated as independent of the spatial resolution. Lower panel: The wall-time $W$ in seconds as a function of price resolution $\Delta S$ for the tests described above. This data is used to fit the scaling law $W \propto \Delta S^{-2 \psi}$ and is almost identical to the data presented for the European case in figure 4. At moderate to high resolution the law is clearly well obeyed. Reference lines scaling with $\Delta S^{-1}$ and $\Delta S^{-2}$ are also shown.

pricing American options, for example Nielsen et al. (2002). Power law indices are fit to the well-behaved datapoints according to $W \propto \Delta S^{\psi}$ and tabulated in table 2 . Reference lines are plotted for some values in the corresponding figure panels.

Clearly, from the upper panel of figure 4, the temporal accuracy of all schemes is independent of the mesh spacing in price.

\subsection{Wall-time scaling with temporal resolution: determining $\alpha$}

We now proceed to establish the values of $\alpha$ using equations (40) and (41). The temporal error $E_{t}$ and 

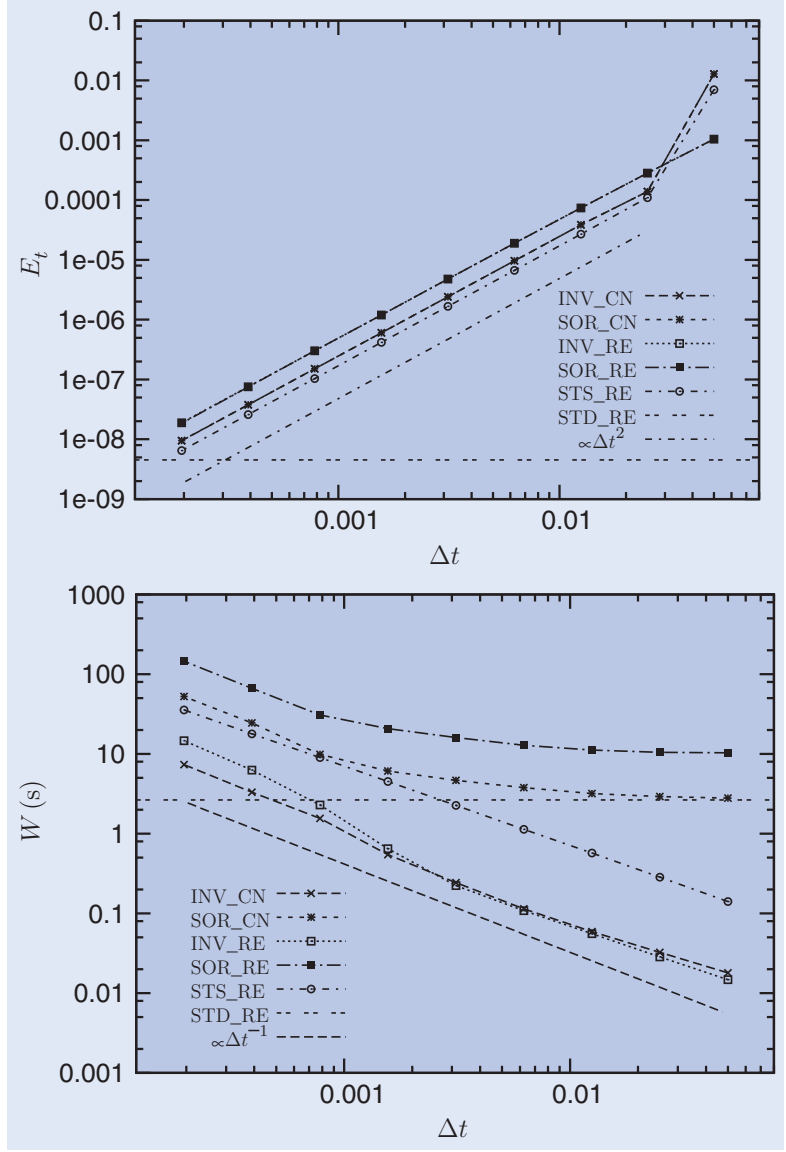

Figure 6. Temporal error and wall-time scaling with time resolution for European put option pricing. Temporal error $E_{t}$ at the strike price (upper panel), and wall-time $W$ in seconds (lower panel) plotted against $\Delta t$ for a fixed value of $\Delta S=5 \mathrm{~K} /$ 500. Reference value are indicated for STD RE with $\Delta t=10^{-4}$ as it is unstable over the plotted range of $\Delta t$. Errors are calculated using a high-accuracy approximation to the exact solution of equation (11) obtained from an independent code modified to be second order in time (Ødegaarde 2007). This data from the upper panel is used to fit $\beta$ to the scaling law $\beta=\alpha \zeta^{-1 / p} E_{S}^{-\psi}$. Reference lines illustrating the approximate scaling laws $E_{t} \propto \Delta t^{2}$ and $W \propto \Delta t^{-1}$ are presented in the upper and lower panels, respectively. Note that $p=2$ in all cases.

wall-time $W$ are plotted in figures 6 and 7 for the European and American option pricing problems, respectively. A range of temporal resolutions is considered, $\Delta t=\left\{T /\left(20 \times 2^{n}\right) \mid n=0,1, \ldots, 7,8\right\}$, for the single spot price spacing value $\Delta S=1$. The upper panel in each case shows the temporal error $E_{t}$ while the lower panel illustrates the wall-time $W$. STD_RE is not stable for any values of $\Delta t$ in this range and so a reference value is plotted for the minimal stable value of $\Delta t=10^{-4}$. Table 3 explicitly provides all the errors and timings for this set of tests as well as the benchmark values from which $E_{S}=\left|V(K, 0)-V_{\Delta S}(K, 0)\right|$ is derived.

From the plots of $E_{t}$ in figures 6 and 7, the temporal order of accuracy, $p$, for each scheme is 2, except for INV_CN in the American option pricing case where $p=1$, as has already been confirmed. Again, the early exercise constraint results in less smooth convergence
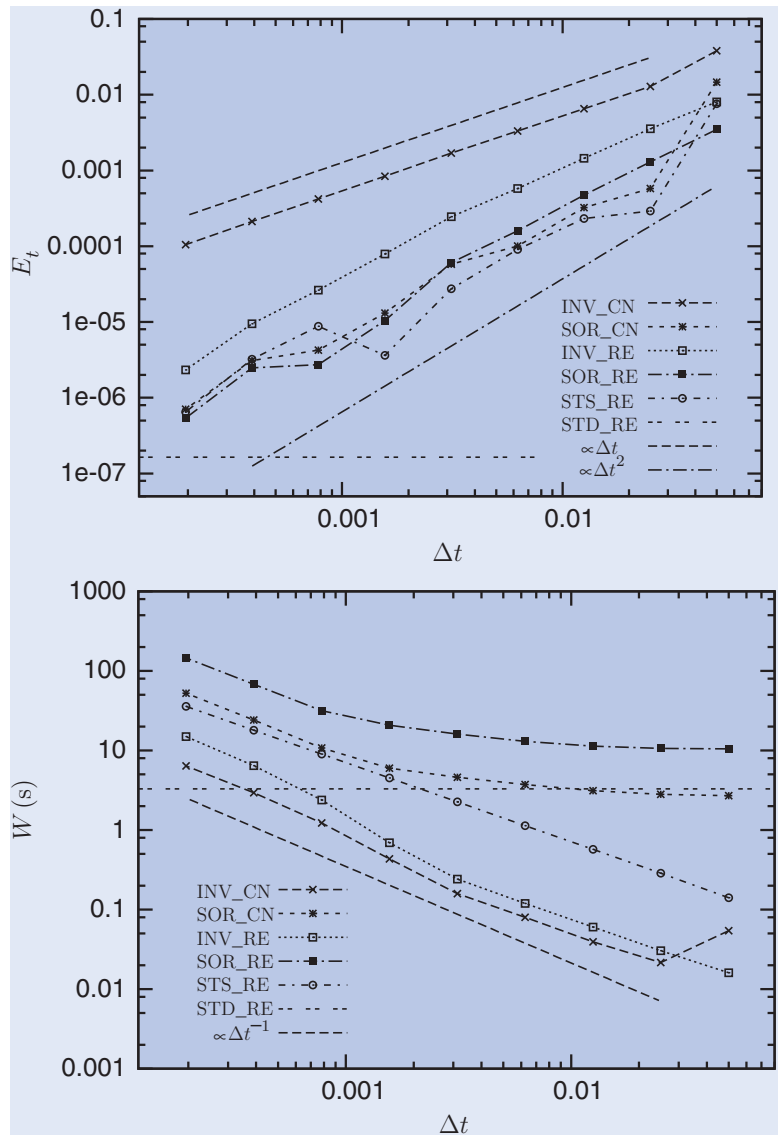

Figure 7. Temporal error and wall-time scaling with time resolution for American put option pricing. Temporal error $E_{t}$ at the strike price (upper panel), and wall-time $W$ in seconds (lower panel) plotted against $\Delta t$ for a fixed value of $\Delta S=5 \mathrm{~K} /$ 500. Reference value are indicated for STD_RE with $\Delta t=10^{-4}$ as it is unstable over the plotted range of $\Delta t$. Errors are calculated using a high-accuracy approximation to the exact solution of equation (11) obtained from an independent code modified to be second order in time (Ødegaarde 2007). This data from the upper panel is used to fit $\beta$ to the scaling law $\beta=\alpha \zeta^{-1 / p} E_{S}^{-\psi}$. Reference lines illustrating the approximate scaling laws $E_{t} \propto \Delta t^{p}$ and $W \propto \Delta t^{-1}$ are presented in the upper and lower panels, respectively. Note that $p=2$ in all cases except for INV_CN where $p=1$.

Table 1. Additional semi-empirical efficiency function parameters for CFL condition compliance of explicit schemes: $(\epsilon, \zeta, \eta)$.

\begin{tabular}{lcccccccc}
\hline & \multicolumn{3}{c}{ European } & & \multicolumn{3}{c}{ American } \\
\cline { 2 - 4 } \cline { 6 - 8 } Method & $\epsilon$ & $\zeta$ & $\eta$ & & $\zeta$ & $\eta$ \\
\hline STS_RE & 0.0591 & 0.1791 & 0.0025 & & 0.0591 & 0.4368 & 0.0031 \\
STD_RE & 0.0001 & 0.4787 & 0.0025 & & 0.0001 & 1.7397 & 0.0031 \\
\hline
\end{tabular}

Table 2. Parameters for semi-empirical efficiency functions: $(\alpha, \psi, p)$.

\begin{tabular}{|c|c|c|c|c|c|c|}
\hline \multirow[b]{2}{*}{ Method } & \multicolumn{3}{|c|}{ European } & \multicolumn{3}{|c|}{ American } \\
\hline & $\alpha$ & $\psi$ & $p$ & $\alpha$ & $\psi$ & $p$ \\
\hline NV_CN & $5.1672 \mathrm{e}-07$ & 1.1361 & 2 & $4.1479 \mathrm{e}-07$ & 1.1266 & 1 \\
\hline SOR_CN & $5.6559 \mathrm{e}-06$ & 1.1188 & 2 & $7.6095 e-06$ & 1.0978 & 2 \\
\hline INV_RE & $6.3219 \mathrm{e}-07$ & 1.1852 & 2 & $2.5394 \mathrm{e}-06$ & 1.1607 & 2 \\
\hline SOR_RE & $1.9042 \mathrm{e}-05$ & 1.1539 & 2 & $4.0585 e-05$ & 1.1497 & 2 \\
\hline STS_RE & $2.6033 \mathrm{e}-04$ & 0.4063 & 2 & $4.2956 \mathrm{e}-04$ & 0.4078 & 2 \\
\hline STD_RE & $1.9161 \mathrm{e}-05$ & 0.3758 & 2 & $4.8678 \mathrm{e}-05$ & 0.3740 & 2 \\
\hline
\end{tabular}


Table 3. Numerical value $V(K)$ at strike price $S=K$ with wall-time $W$ in seconds, and temporal error $E_{t}$ derived from high-accuracy benchmark value (denoted Bench). $\Delta S=5 K / 500$ in all cases. Exact solution for European put option obtained from Black-Scholes equation (denoted BS) or high-accuracy solution for American put option derived from binomial tree method (denoted BT) also shown for reference.

\begin{tabular}{|c|c|c|c|c|c|c|c|}
\hline \multirow[b]{2}{*}{$N$} & \multirow[b]{2}{*}{ Method } & \multicolumn{3}{|c|}{ European put } & \multicolumn{3}{|c|}{ American put } \\
\hline & & $V(K)$ & $W(\mathrm{~s})$ & $E_{t}$ & $V(K)$ & $W(\mathrm{~s})$ & $E_{t}$ \\
\hline & $\begin{array}{l}\text { BS/BT } \\
\text { Bench }\end{array}$ & $\begin{array}{l}5.5735260222 \\
5.5710548584\end{array}$ & & & $\begin{array}{l}6.0903702250 \\
6.0874933186\end{array}$ & & \\
\hline 20 & $\begin{array}{l}\text { INV_CN } \\
\text { SOR_CN } \\
\text { INV_RE } \\
\text { SOR_RE } \\
\text { STS_RE }\end{array}$ & $\begin{array}{l}5.5582337988 \\
5.5582337980 \\
5.5700055968 \\
5.5700055955 \\
5.5640341499\end{array}$ & $\begin{array}{r}0.0180 \\
2.7834 \\
0.0147 \\
10.3012 \\
0.1405\end{array}$ & $\begin{array}{l}1.3 \mathrm{e}-02 \\
1.3 \mathrm{e}-02 \\
1.0 \mathrm{e}-03 \\
1.0 \mathrm{e}-03 \\
7.0 \mathrm{e}-03\end{array}$ & $\begin{array}{l}6.0494286083 \\
6.0728821885 \\
6.0794452317 \\
6.0840121118 \\
6.0799065958\end{array}$ & $\begin{array}{r}0.0543 \\
2.7081 \\
0.0160 \\
10.4513 \\
0.1407\end{array}$ & $\begin{array}{l}3.8 \mathrm{e}-02 \\
1.5 \mathrm{e}-02 \\
8.0 \mathrm{e}-03 \\
3.5 \mathrm{e}-03 \\
7.6 \mathrm{e}-03\end{array}$ \\
\hline 40 & $\begin{array}{l}\text { INV_CN } \\
\text { SOR_CN } \\
\text { INV_RE } \\
\text { SOR_RE } \\
\text { STS_RE }\end{array}$ & $\begin{array}{l}5.5711933839 \\
5.5711933832 \\
5.5707713289 \\
5.5707713279 \\
5.5709447761\end{array}$ & $\begin{array}{r}0.0326 \\
2.9173 \\
0.0284 \\
10.4519 \\
0.2850\end{array}$ & $\begin{array}{l}1.4 \mathrm{e}-04 \\
1.4 \mathrm{e}-04 \\
2.8 \mathrm{e}-04 \\
2.8 \mathrm{e}-04 \\
1.1 \mathrm{e}-04\end{array}$ & $\begin{array}{l}6.0746091394 \\
6.0869159940 \\
6.0839310254 \\
6.0861990167 \\
6.0872009272\end{array}$ & $\begin{array}{r}0.0216 \\
2.8169 \\
0.0304 \\
10.6100 \\
0.2859\end{array}$ & $\begin{array}{l}1.3 e-02 \\
5.8 e-04 \\
3.6 e-03 \\
1.3 e-03 \\
2.9 e-04\end{array}$ \\
\hline 80 & $\begin{array}{l}\text { INV_CN } \\
\text { SOR_CN } \\
\text { INV_RE } \\
\text { SOR_RE } \\
\text { STS_RE }\end{array}$ & $\begin{array}{l}5.5710935500 \\
5.5710935496 \\
5.5709809065 \\
5.5709809058 \\
5.5710278176\end{array}$ & $\begin{array}{r}0.0593 \\
3.1840 \\
0.0559 \\
11.1796 \\
0.5702\end{array}$ & $\begin{array}{l}3.9 \mathrm{e}-05 \\
3.9 \mathrm{e}-05 \\
7.4 \mathrm{e}-05 \\
7.4 \mathrm{e}-05 \\
2.7 \mathrm{e}-05\end{array}$ & $\begin{array}{l}6.0809446348 \\
6.0871705422 \\
6.0860502803 \\
6.0870218427 \\
6.0872609232\end{array}$ & $\begin{array}{r}0.0393 \\
3.1208 \\
0.0601 \\
11.3238 \\
0.5698\end{array}$ & $\begin{array}{l}6.5 \mathrm{e}-03 \\
3.2 \mathrm{e}-04 \\
1.4 \mathrm{e}-03 \\
4.7 \mathrm{e}-04 \\
2.3 \mathrm{e}-04\end{array}$ \\
\hline 160 & $\begin{array}{l}\text { INV_CN } \\
\text { SOR_CN } \\
\text { INV_RE } \\
\text { SOR_RE } \\
\text { STS_RE }\end{array}$ & $\begin{array}{l}5.5710645318 \\
5.5710645311 \\
5.5710359539 \\
5.5710359531 \\
5.5710481552\end{array}$ & $\begin{array}{r}0.1140 \\
3.7710 \\
0.1083 \\
12.8102 \\
1.1305\end{array}$ & $\begin{array}{l}9.7 \mathrm{e}-06 \\
9.7 \mathrm{e}-06 \\
1.9 \mathrm{e}-05 \\
1.9 \mathrm{e}-05 \\
6.7 \mathrm{e}-06\end{array}$ & $\begin{array}{l}6.0841716547 \\
6.0873924444 \\
6.0869151135 \\
6.0873331963 \\
6.0875839962\end{array}$ & $\begin{array}{r}0.0801 \\
3.7226 \\
0.1193 \\
12.9760 \\
1.1340\end{array}$ & $\begin{array}{l}3.3 \mathrm{e}-03 \\
1.0 \mathrm{e}-04 \\
5.8 \mathrm{e}-04 \\
1.6 \mathrm{e}-04 \\
9.1 \mathrm{e}-05\end{array}$ \\
\hline 320 & $\begin{array}{l}\text { INV_CN } \\
\text { SOR_CN } \\
\text { INV_RE } \\
\text { SOR_RE } \\
\text { STS_RE }\end{array}$ & $\begin{array}{l}5.5710572768 \\
5.5710572766 \\
5.5710500779 \\
5.5710500788 \\
5.5710531897\end{array}$ & $\begin{array}{r}0.2433 \\
4.6564 \\
0.2224 \\
16.0405 \\
2.2636\end{array}$ & $\begin{array}{l}2.4 \mathrm{e}-06 \\
2.4 \mathrm{e}-06 \\
4.8 \mathrm{e}-06 \\
4.8 \mathrm{e}-06 \\
1.7 \mathrm{e}-06\end{array}$ & $\begin{array}{l}6.0858018523 \\
6.0874354049 \\
6.0872476891 \\
6.0874324039 \\
6.0874658348\end{array}$ & $\begin{array}{r}0.1582 \\
4.6028 \\
0.2408 \\
16.0467 \\
2.2593\end{array}$ & $\begin{array}{l}1.7 e-03 \\
5.8 e-05 \\
2.5 e-04 \\
6.1 e-05 \\
2.7 e-05\end{array}$ \\
\hline 640 & $\begin{array}{l}\text { INV_CN } \\
\text { SOR_CN } \\
\text { INV_RE } \\
\text { SOR_RE } \\
\text { STS_RE }\end{array}$ & $\begin{array}{l}5.5710554631 \\
5.5710554630 \\
5.5710536564 \\
5.5710536564 \\
5.5710544422\end{array}$ & $\begin{array}{r}0.5446 \\
6.0882 \\
0.6477 \\
20.7108 \\
4.4999\end{array}$ & $\begin{array}{l}6.0 \mathrm{e}-07 \\
6.0 \mathrm{e}-07 \\
1.2 \mathrm{e}-06 \\
1.2 \mathrm{e}-06 \\
4.2 \mathrm{e}-07\end{array}$ & $\begin{array}{l}6.0866566365 \\
6.0874801971 \\
6.0874145168 \\
6.0874829213 \\
6.0874969531\end{array}$ & $\begin{array}{r}0.4338 \\
5.9971 \\
0.6917 \\
20.7715 \\
4.5181\end{array}$ & $\begin{array}{l}8.4 \mathrm{e}-04 \\
1.3 \mathrm{e}-05 \\
7.9 \mathrm{e}-05 \\
1.0 \mathrm{e}-05 \\
3.6 \mathrm{e}-06\end{array}$ \\
\hline 1280 & $\begin{array}{l}\text { INV_CN } \\
\text { SOR_CN } \\
\text { INV_RE } \\
\text { SOR_RE } \\
\text { STS_RE }\end{array}$ & $\begin{array}{l}5.5710550096 \\
5.5710550091 \\
5.5710545571 \\
5.5710545561 \\
5.5710547545\end{array}$ & $\begin{array}{r}1.5557 \\
9.8862 \\
2.2893 \\
30.8410 \\
9.0119\end{array}$ & $\begin{array}{l}1.5 \mathrm{e}-07 \\
1.5 \mathrm{e}-07 \\
3.0 \mathrm{e}-07 \\
3.0 \mathrm{e}-07 \\
1.0 \mathrm{e}-07\end{array}$ & $\begin{array}{l}6.0870731887 \\
6.0874890604 \\
6.0874669422 \\
6.0874905967 \\
6.0875020780\end{array}$ & $\begin{array}{r}1.2253 \\
10.7403 \\
2.3884 \\
31.6979 \\
9.0130\end{array}$ & $\begin{array}{l}4.2 e-04 \\
4.3 e-06 \\
2.6 e-05 \\
2.7 e-06 \\
8.8 e-06\end{array}$ \\
\hline 2560 & $\begin{array}{l}\text { INV_CN } \\
\text { SOR_CN } \\
\text { INV_RE } \\
\text { SOR_RE } \\
\text { STS_RE }\end{array}$ & $\begin{array}{l}5.5710548963 \\
5.5710548963 \\
5.5710547830 \\
5.5710547835 \\
5.5710548325\end{array}$ & $\begin{array}{r}3.3009 \\
24.5080 \\
6.2863 \\
66.6839 \\
17.8931\end{array}$ & $\begin{array}{l}3.8 \mathrm{e}-08 \\
3.8 \mathrm{e}-08 \\
7.5 \mathrm{e}-08 \\
7.5 \mathrm{e}-08 \\
2.6 \mathrm{e}-08\end{array}$ & $\begin{array}{l}6.0872818618 \\
6.0874902341 \\
6.0874838183 \\
6.0874908470 \\
6.0874965528\end{array}$ & $\begin{array}{r}2.9442 \\
24.0889 \\
6.4247 \\
67.6991 \\
17.9378\end{array}$ & $\begin{array}{l}2.1 \mathrm{e}-04 \\
3.1 \mathrm{e}-06 \\
9.5 \mathrm{e}-06 \\
2.5 \mathrm{e}-06 \\
3.2 \mathrm{e}-06\end{array}$ \\
\hline 5120 & $\begin{array}{l}\text { INV_CN } \\
\text { SOR_CN } \\
\text { INV_RE } \\
\text { SOR_RE } \\
\text { STS_RE }\end{array}$ & $\begin{array}{l}5.5710548679 \\
5.5710548680 \\
5.5710548396 \\
5.5710548399 \\
5.5710548520\end{array}$ & $\begin{array}{r}7.3201 \\
52.3461 \\
14.6579 \\
145.4471 \\
35.6537\end{array}$ & $\begin{array}{l}9.4 \mathrm{e}-09 \\
9.5 \mathrm{e}-09 \\
1.9 \mathrm{e}-08 \\
1.9 \mathrm{e}-08 \\
6.5 \mathrm{e}-09\end{array}$ & $\begin{array}{l}6.0873884923 \\
6.0874926123 \\
6.0874909960 \\
6.0874927814 \\
6.0874926711\end{array}$ & $\begin{array}{r}6.4074 \\
52.5042 \\
14.9043 \\
145.9531 \\
35.9482\end{array}$ & $\begin{array}{l}1.0 e-04 \\
7.1 e-07 \\
2.3 e-06 \\
5.4 e-07 \\
6.5 e-07 e\end{array}$ \\
\hline 10000 & STD_RE & 5.5710548540 & 2.6428 & $4.5 e-09$ & 6.0874931545 & 3.2914 & $1.6 \mathrm{e}-07$ \\
\hline
\end{tabular}

profiles for the American option. Best-fit values for $\beta$ are obtained using equation (41).

High temporal accuracy solutions obtained using the binomial tree method yield $E_{S}=0.0024711638$ for the European option and $E_{S}=0.0028769064$ for the
American option. Then $\alpha=\beta E_{S}^{\psi}$ follows from equation (41). The efficiency parameters $(\alpha, \psi, p)$ are now fully determined and presented in table 2.

The assumption of inverse scaling of $W$ with $\Delta t$ given by equation (36) is supported by the lower panels of 
figures 6 and 7 except for the SOR schemes at low temporal resolution where the convergence properties of these schemes are poor.

\subsection{Stability constraint for explicit schemes: determining $\epsilon$}

As discussed in sections 2.2 and 4.1, explicit schemes are limited by the CFL condition given by equation (26). The critical error, $E_{\text {crit }}$, below which this constraint applies, is derived from equation (45). $\zeta$ and $\eta$, as discussed in section 5.1, have been determined and are presented in table 1. All that remains to characterize the CFL constraint is to establish values for $\epsilon$.

For the cases under consideration here, with $\sigma=0.2$, $S_{\infty}=5 K$, and $K=100$, equation (46) agrees with experiment to within $1 \%$ and we take $\epsilon_{\text {STD_RE }}=$ $\epsilon_{\mathrm{STD} \text { theory }}=10^{-4}$. On the other hand, STS_RE turns out to be particularly well suited to this problem and is stable well beyond the value of $\epsilon_{\text {STS_RE }}^{\text {theory }}=380 \epsilon_{\text {STD_RE }}$ obtained from equation (47) with $N_{\mathrm{STS}}=30$ and $v=5 \times 10^{-4}$. In fact, we find experimentally that $\epsilon_{\mathrm{STS} \_\mathrm{RE}}=591 \epsilon_{\mathrm{STD} \_\mathrm{RE}}$. Presented in table 1 , we now have $(\epsilon, \zeta, \eta)$ as required for the CFL constraint on the explicit schemes STS_RE and STD_RE.

\subsection{Semi-empirical efficiency functions}

We now present the semi-empirical evaluation of the wall-time $W$ in seconds, for each of the six schemes, as a function of the error $E$ for the European and American variants of the option pricing problem under consideration. Equation (43) is valid for all cases except for the explicit schemes below the critical error $E_{\text {crit }}$ (equation (45)) when the appropriate expression becomes equation (49) due to the CFL condition for stability. The parameters $(\alpha, \psi, p)$, required in the former instance, and $(\epsilon, \zeta, \eta)$, as required in the latter instance, are presented in tables 1 and 2 respectively.

The results of the estimated optimal scheme wall-times are plotted in figure 8 in the range $10^{-6} \leq E \leq 10^{-2}$. The break in the power law can clearly be seen for STS_RE at $E_{\text {crit }}=0.0269$ in the European option plot and at 0.0168 for the American case. No such break is visible for the STD_RE lines because $E_{\text {crit }}>10^{3}$ in both cases: well above the maximum plotted error value.

Before interpreting these results it is important to emphasize that the results are biased heavily in favour of the implicit schemes. In particular, for the direct matrix inversion methods, the necessary matrix inversions are carried out only once before the timer is started. The inversions are usually slower than the time integration itself for $\Delta S \ll 1$, and, in general, the coefficients may be time varying and therefore this procedure will be required at each time-step. Secondly, for the SOR methods at $\Delta t \gtrsim 10^{-3}$, the wall-time to convergence plateaus at a value in excess of the assumed power law form.

Even with these advantages, STS_RE is clearly the optimal performer when taken as a broad spectrum method for this problem. At all but low accuracies for

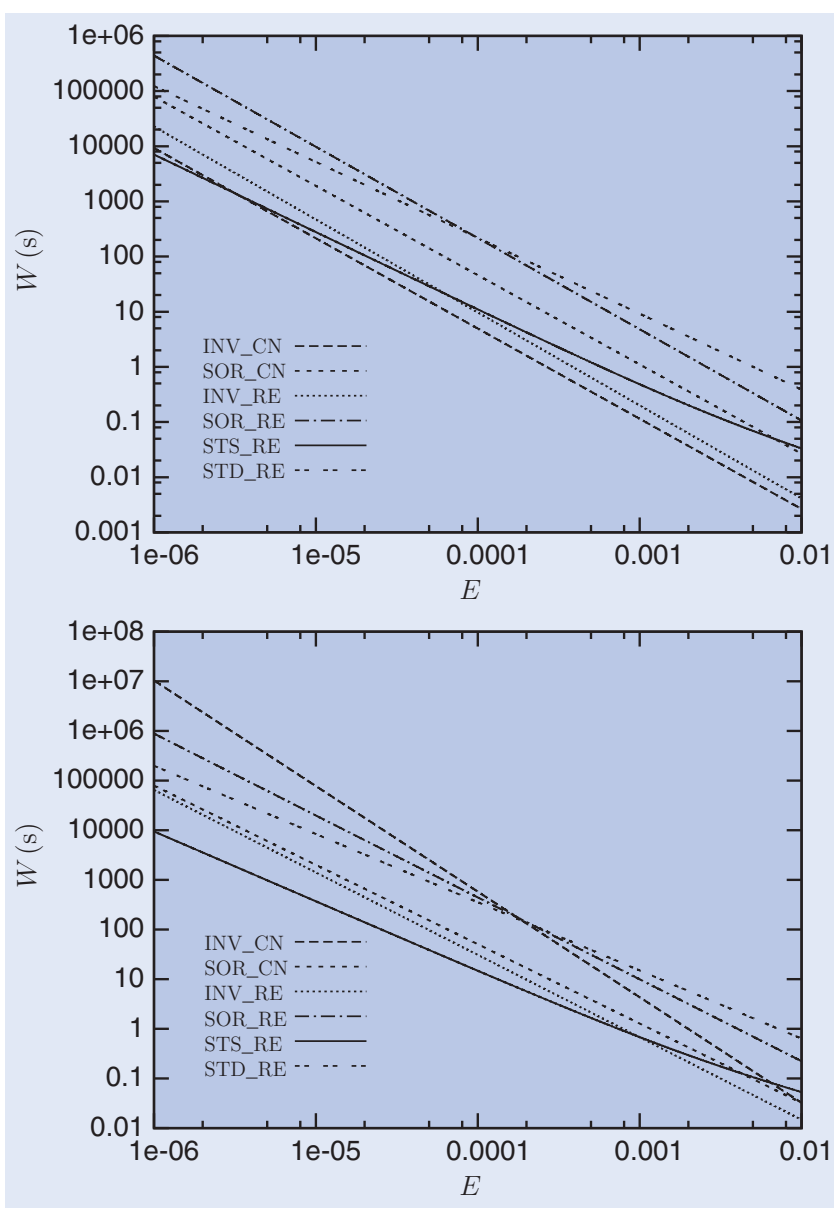

Figure 8. Evaluation of semi-empirical efficiency functions for European and American put options with $\sigma=0.2, r=0.05$, $T=1.0, S_{\infty}=5 K$, and $K=100$. Wall-time $W$ in seconds is plotted against required total error $E$ in the solution for value at strike price $V(K)$. Upper panel: European put option. For $E \gtrsim 10^{-4}$, STS_RE is inferior only to the direct matrix inversion methods, otherwise it is equivalent or superior. Note the break at $E_{\text {crit }}=0.0269$ in the case of STS_RE below which the optimal relationship between the spatial and temporal errors, $E_{S}$ and $E_{t}$, is constrained by the CFL condition. Lower panel: American put option. In this test, STS_RE is superior for $E \lesssim 10^{-2}$. Note the break at $E_{\text {crit }}=0.0168$ for STS_RE.

some of the alternative implicit methods where wall-time is low in any case, it is the most efficient option. When the simplicity of the method and the above provisos are taken into consideration, the case for using STS_RE as opposed to any of the presented implicit methods is overwhelming.

\section{Conclusion}

An acceleration technique, known as Super-TimeStepping (STS), for explicit finite difference algorithms is introduced for the first time in computational finance. We demonstrate the efficacy of the method by pricing European and American put options in a series of benchtests with several well-known finite difference techniques. Simple vanilla options are chosen as case studies for their inherent simplicity. However, in practice, the target application for this method is the numerical modeling 
of more complex systems for which implicit methods quickly become prohibitively difficult to implement. For example, we suggest multi-dimensional systems requiring decomposed and/or adaptive meshes. Stability is formally demonstrated for a novel operator split implementation of the technique. This composite method is of greater generality and it is suggested that it may be the appropriate approach when considering pricing of more exotic financial instruments.

A novel methodology to assess and compare the schemes' efficiencies is also introduced. Applying this technique to test cases we demonstrate degrees of acceleration provided by the STS method which yield comparable, and even superior, efficiencies to implicit differencing methods. The implicit methods considered are PSOR and direct matrix inversion in both European and American cases. Of central importance, this is achieved with no significant increase in implementation complexity over and above that of the underlying standard explicit algorithm.

Given that STS accelerated methods inherit the simplicity of explicit methods whilst achieving high accuracy at low computational cost, we conclude that when faced with complex pricing models this approach offers a highly attractive alternative to the substantial challenges presented by conventional implicit techniques. Promising targets include models involving multidimensional parameter spaces, variable meshes, or moving boundaries.

\section{Acknowledgements}

The authors are grateful to the UCD Complex and Adaptive Systems Laboratory for facilitating this collaboration.

\section{References}

Alexiades, V., Amiez, G. and Gremaud, P., Super-TimeStepping acceleration of explicit schemes for parabolic problems. Com. Numer. Meth. Engng., 1996, 12, 31-42.

Black, F. and Scholes, M.S., The pricing of options and corporate liabilities. J. Polit. Econ., 1973, 81, 637-654.

Brennan, M. and Schwartz, E., The valuation of American put options. J. Finance, 1977, 32, 449-462.

Broadie, M. and Detemple, J., American option valuation: new bounds, approximations, and a comparison of existing methods. Rev. Financial Stud., 1996, 9, 1211-1259.

Crank, J., Free and Moving Boundary Problems, 1984 (Clarendon Press: Oxford).

Duffie, D., Dynamic Asset Pricing Theory, 1996 (Princeton University Press: Princeton).

Evje, S., Karlsen, K.H. and Risebro, N.H., A continuous dependence result for nonlinear degenerate parabolic equations with spatially dependent flux function. In Hyperbolic Problems: Theory, Numerics, Applications, edited by H. Freistüler and G. Warnecke, pp. 337-346, 2001 (Birkhäuser).

Gentzsch, W., Numerical solution of linear and non-linear parabolic differential equations by a time-discretisation of third order accuracy. In Proceeding of the Third GAMM Conference on Numerical Methods in Fluid Mechanics, edited by E.H. Hirschel, pp. 109-117, 1979 (Friedr. Vieweg \& Sohn).
Gerschgorin, S., Über die Abgrenzung der Eigenwerte einer Matrix. Izv. Akad. Nauk SSSR, Ser. Mat., 1931, 7, 749-754.

Geske, R. and Johnson, H.E., The American put option valued analytically. J. Finance, 1984, 39, 1511-1524.

Hager, W.W., Applied Numerical Linear Algebra, 1988 (Prentice-Hall: Englewood Cliffs, NJ).

Ikonen, S. and Toivanen, J., Pricing American options using LU decomposition. Appl. Math. Sci., 2007a, 1, 2529-2551.

Ikonen, S. and Toivanen, J., Efficient numerical methods for pricing American options under stochastic volatility. Numer. Meth. Partial Differ. Eqns, 2007b, 24, 104-126.

Markoff, W., Über Polynome, die in einem gegebenen Intervalle möglichst wenig von null abweichen. Math. Ann., 1916, 77, 213-258, translated by J. Grossman from original Russian article published in 1892 .

Merton, R.C., The theory of rational option pricing. Bell J. Econ. Mgmt Sci., 1973, 4, 141-183.

Mignone, A., Bodo, G., Massaglia, S., Matsakos, T., Tesileanu, O., Zanni, C. and Ferrari, A., PLUTO: a numerical code for computational astrophysics. ApJS, 2007, 170, 228.

Morton, K.W., Stability of finite difference approximations to a diffusion-convection equation. Int. J. Numer. Meth. Engng, 1980, 15, 677-683.

Nielsen, B., Skavhaug, O. and Tveito, A., Penalty and frontfixing methods for the numerical solution of American option problems. J. Comput. Finance, 2002, 5, 69-97.

Ødegaarde, B.A., Financial numerical recipes in $\mathrm{C}++, 2007$. Available online at: http://finance.bi.no/ bernt/gcc_prog/ recipes/recipes.pdf

O'Sullivan, S. and Downes, T.P., An explicit scheme for multifluid magnetohydrodynamics. MNRAS, 2006, 366, 1329.

O'Sullivan, S. and Downes, T.P., A three-dimensional numerical method for modelling weakly ionized plasmas. MNRAS, 2007, 376, 1648.

Sbalzarini, I.F., Hayer, A., Helenius, A. and Koumoutsakos, P., Simulations of (an)isotropic diffusion on curved biological surfaces. Biophys. J., 2006, 90, 8478-8485.

Shi, Y., Li, L. and Liang, C.H., Multidomain pseudospectral time-domain algorithm based on super-time-stepping method. IEE Proc. Microwaves, Antennas Propagation, 2006, 153, 55-60.

Sommeijer, B.P., Shampine, L.F. and Verwer, J.G., RKC: An explicit solver for parabolic PDEs. Technical Report MASR9715, CWI Amsterdam, 1997.

Tavella, D. and Randall, C., Pricing Financial Instruments: The Finite Difference Method, 2000 (Wiley: New York).

van der Houwen, P.J., Construction of Integration Formulas for Initial Value Problems, 1977 (North-Holland: Amsterdam).

van der Houwen, P.J. and Sommeijer, B.P., On the internal stability of explicit $m$-stage Runge-Kutta methods for large values of $m$. Z. Angew. Math. Mech., 1980, 60, 479-485.

Verwer, J.G., Explicit Runge-Kutta methods for parabolic partial differential equations. Appl. Numer. Math., 1996, 22, 359-379.

Verwer, J.G., Hundsdorfer, W.H. and Sommeijer, B.P., Convergence properties of the Runge-Kutta-Chebyshev method. Numer. Math., 1990, 57, 157-178.

Wilmott, P., Howison, S. and Dewynne, J., Option Pricing: Mathematical Models and Computation, 1995 (Oxford Financial Press: Oxford).

Zhu, S.P., An exact and explicit solution for the valuation of American put options. Quant. Finance, 2006, 6, 229-242.

\section{Appendix A: Composite acceleration method}

The fully explicit $(\theta=1)$ expression of equation (22) is

$$
\mathbf{V}^{n}=(\mathbf{I}-\Delta t \mathbf{P}) \mathbf{V}^{n+1}-\Delta t \mathbf{b}^{n+1},
$$

with all quantities defined as in section 2.2 . 
Although in sample cases STS applied to a nonsymmetric operator has been shown to result in a slight flattening of the stability region (Alexiades et al. 1996), formally stability may only be established for the symmetric positive definite case. For the problems considered in the present work, we have found that STS may be applied to the weakly non-symmetric matrix $\mathbf{P}$ with negligible impact on the scheme's stability. For completeness, however, we now proceed to determine conditions for strict stability of an alternate finite differencing of the Black-Scholes equation which does incorporate a symmetric positive definite operator. This scheme formally admits application of the STS acceleration method.

$\mathbf{P}$ may be trivially decomposed according to

$$
\mathbf{P}=\mathbf{A}+\mathbf{K},
$$

where $\mathbf{A} \equiv\left(\mathbf{P}+\mathbf{P}^{\mathrm{T}}\right) / 2$ and $\mathbf{K} \equiv\left(\mathbf{P}-\mathbf{P}^{\mathrm{T}}\right) / 2$ are the symmetric and skew-symmetric parts of $\mathbf{P}$, respectively, with $\cdot{ }^{\mathrm{T}}$ denoting a transpose operation. Explicitly, these matrices are given by

$$
\mathbf{A}=\left[\begin{array}{cccc}
b_{1} & \frac{1}{2}\left(a_{2}+c_{1}\right) & \cdots & 0 \\
\vdots & \ddots & \ddots & \vdots \\
0 & \cdots & \frac{1}{2}\left(a_{J-1}+c_{J-2}\right) & b_{J-1}
\end{array}\right]
$$

and

$$
\mathbf{K}=\left[\begin{array}{cccc}
0 & -\frac{1}{2}\left(a_{2}-c_{1}\right) & \cdots & 0 \\
\vdots & \ddots & \ddots & \vdots \\
0 & \cdots & \frac{1}{2}\left(a_{J-1}-c_{J-2}\right) & 0
\end{array}\right] .
$$

Prescribing the update of $\mathbf{V}^{n}$ to $\mathbf{V}^{n+1}$ via Multiplicative Operator Splitting (MOS) we write

$$
\mathbf{V}^{n}=\mathbf{R} \mathbf{T} \mathbf{V}^{n+1}-\Delta t \mathbf{b}^{n+1} .
$$

Strict stability requires that $\|\mathbf{R T}\| \leq 1$ for any operator norm (Morton 1980). By multiplicative dominance $\|\mathbf{R T}\| \leq\|\mathbf{R}\|\|\mathbf{T}\|$. For stability we therefore demand $\|\mathbf{R}\| \leq 1$ and $\|\mathbf{T}\| \leq 1$ for operators $\mathbf{R}$ and $\mathbf{T}$ consistent with equation (A1). We shall adopt the Euclidean operator norm, denoted $\|\cdot\|_{2}$, hereafter.

Note that, for any complex square matrix $\mathbf{X}$, we may write $\|\mathbf{X}\|_{2}=\sqrt{\rho\left(\mathbf{X}^{\dagger} \mathbf{X}\right)}$, where $\mathbf{X}^{\dagger}$ denotes the complex conjugate of $\mathbf{X} \in \mathbb{C}^{n} \times \mathbb{C}^{n}$ and $\rho(\cdot)$ indicates the spectral radius of an operator (i.e. the maximum of the absolute values of the operator's eigenvalues).

We propose $\mathbf{R}=\mathbf{I}-\Delta t \mathbf{A}$, which is symmetric by construction and clearly consistent with equation (A1). We now proceed to establish the positive definiteness and stability properties for this definition.

By Sylvester's criterion, a matrix is positive definite if and only if the determinants of all upper-left sub-matrices are positive. The determinant, $D_{j}$, for the upper-left $j \times j$ sub-matrix of the tri-diagonal matrix (23) is easily expressed by means of a simple three-term recurrence relation (e.g., Hager 1988)

$$
D_{j}=b_{j} D_{j-1}-\frac{1}{4}\left(a_{j}+c_{j-1}\right)^{2} D_{j-2},
$$

with $D_{-1} \equiv 0$ and $D_{0} \equiv 1$. It is straightforward to show that

$$
D_{j}=\sum_{k=0}^{j} d_{j k} \sigma^{2 k} r^{j-k},
$$

where $d_{j k}$ is a positive real number for all values of $k$.

Under the condition $r>0, \mathbf{A}$ is therefore strictly positive-definite. Note that this result does not depend on uniform $\sigma(S)$ and $r(S)$. Incidentally, this result implies that $\mathbf{P}$ must be positive stable.

In order to determine the stability limit $\Delta t_{\mathrm{STD}}$, Gerschgorin's (1931) theorem may be invoked. The eigenvalues $\lambda_{j}^{A}$ of $\mathbf{A}$ are bounded by the relation

$$
\left|\lambda_{j}^{A}-b_{j}\right| \leq\left|\frac{1}{2}\left(a_{j}+c_{j-1}\right)\right|+\left|\frac{1}{2}\left(a_{j+1}+c_{j}\right)\right|,
$$

for $j=\{1, \ldots, J-1\}$.

Under the usual assumption of $\sigma^{2} S_{\infty} / r \gg 1$, we find $\lambda_{\max }^{A} \leq 2 \sigma^{2} S_{\infty}^{2} / \Delta S^{2}$. Therefore, since $\mathbf{A}$ is symmetric positive definite we have $\|\mathbf{R}\|_{2}=\sqrt{\rho\left(\mathbf{R}^{2}\right)}=1-\Delta t \lambda_{\max }^{A}$. The resultant stability constraint is again given by equation (26), i.e. $\Delta t \leq \Delta S^{2} / \sigma^{2} S_{\infty}^{2}$.

We now require a suitable prescription for $\mathbf{T}$ to complete the definition of the scheme. The simple choice of $\mathbf{T}=\mathbf{I}-\Delta t \mathbf{K}$ is unconditionally unstable since the eigenvalues of any skew symmetric real matrix are purely imaginary (or zero).

However, applying an update derived from a full-step predictor for a skew-symmetric operator is known to retrieve conditional stability for explicit schemes (O'Sullivan and Downes 2006, 2007), i.e. $\mathbf{T V}^{n+1}=$ $\mathbf{V}^{n+1}-\Delta t \mathbf{K} \hat{\mathbf{V}}^{n+1}$ where $\hat{\mathbf{V}}^{n+1}=(\mathbf{I}-\Delta t \mathbf{K}) \mathbf{V}^{n+1}$.

Hence, we adopt the following prescription for $\mathbf{T}$ :

$$
\mathbf{T}=\mathbf{I}-\Delta t \mathbf{K}+\Delta t^{2} \mathbf{K}^{2} .
$$

We proceed by establishing the stability properties of $\mathbf{T}$.

Since $\mathbf{K}$ is real and skew symmetric, we have $\mathbf{T}^{\dagger} \mathbf{T}=$ $\left(\mathbf{I}+\Delta t \mathbf{K}+\Delta t^{2} \mathbf{K}^{2}\right)\left(\mathbf{I}-\Delta t \mathbf{K}+\Delta t^{2} \mathbf{K}^{2}\right)=\mathbf{I}+\Delta t^{2} \mathbf{K}^{2}+\Delta t^{4} \mathbf{K}^{4}$. Furthermore, $\mathbf{K}$ is normal and may therefore be represented via the relation $\mathbf{K}=\mathbf{U}^{\dagger} \boldsymbol{\Lambda}^{K} \mathbf{U}$ where $\mathbf{U} \in \mathbb{C}^{J-1} \times \mathbb{C}^{J-1}$ is unitary and $\boldsymbol{\Lambda}^{K} \in \mathbb{C}^{J-1} \times \mathbb{C}^{J-1}$ is a diagonal matrix formed from the eigenvalues of $\mathbf{K}$, which occur in imaginary conjugate pairs $\lambda^{K}= \pm i \hat{\lambda}^{K}$ (plus 0 if the dimension is an odd integer). Now, $\mathbf{T}^{\dagger} \mathbf{T}=$ $\mathbf{U}^{\dagger}\left(\mathbf{I}+\Delta t^{2}\left(\boldsymbol{\Lambda}^{K}\right)^{2}+\Delta t^{4}\left(\boldsymbol{\Lambda}^{K}\right)^{4}\right) \mathbf{U}$ and therefore $\|\mathbf{T}\|_{2}=$ $\left[\left(1-\Delta t^{2}\left(\hat{\lambda}^{K}\right)^{2}+\Delta t^{4}\left(\hat{\lambda}^{K}\right)^{4}\right)_{\max }\right]^{1 / 2}$ since $\|\mathbf{U}\|_{2}=1$. We apply Gerschgorin's theorem to establish that $\left|\hat{\lambda}^{K}\right|_{\max } \leq\left|\sigma^{2}-r\right| S_{\infty} / \Delta S$ and hence the condition for stability $\Delta t \leq \Delta S /\left|\sigma^{2}-r\right| S_{\infty}$. This will in general be an insignificant constraint with respect to equation (26).

The conditionally stable split scheme is therefore

$$
\mathbf{V}^{n}=\left(\mathbf{I}-\Delta t \mathbf{A}\left(\mathbf{I}-\Delta t \mathbf{K}+\Delta t^{2} \mathbf{K}^{2}\right) \mathbf{V}^{n+1}-\Delta t \mathbf{b}^{n+1} .\right.
$$

Since $\mathbf{A}$ is proven positive definite, acceleration via STS is formally admissible with the inner sub-steps, $\Delta t_{j}$, derived from the normal explicit time-step limit $\Delta t_{\text {STD }}$ using equation (31). In particular, recall that $\Delta t_{\mathrm{STS}} \rightarrow$ $N_{\mathrm{STS}}^{2} \Delta t_{\mathrm{STD}}$ as $v \rightarrow 0$ where $v$ is a user-defined damping 
parameter, and $\Delta t_{\mathrm{STS}}=\sum_{j=1}^{N_{\mathrm{STS}}} \Delta t_{j}$. The full accelerated scheme may therefore be written as

$$
\mathbf{V}^{n}=\left(\prod_{j=1}^{N_{\mathrm{STS}}}\left(\mathbf{I}-\Delta t_{j} \mathbf{A}\right)\right)\left(\mathbf{I}-\Delta t \mathbf{K}+\Delta t^{2} \mathbf{K}^{2}\right) \mathbf{V}^{n+1}-\Delta t \mathbf{b}^{n+1},
$$

(A11)

with the usual CFL condition $\Delta t \leq \Delta S^{2} / \sigma^{2} S_{\infty}^{2}$.

In summary, via MOS of the base scheme (A1), we have established strict stability of the consistent scheme (A11) under the usual time-step constraint (26). However, in this case, STS is formally admissible and yields acceleration in line with the results demonstrated in the main body of this work. In trials, we find that for the tests presented in this paper, the scheme (A11) yields negligible difference from the unsplit case (A1). This is in agreement with other authors who have noted in test cases that relaxing the symmetric positive definite requirement for the application of STS results only in a slight reduction of the stability region in the complex plane along the imaginary direction as long as the evolution operator is only weakly non-symmetric. 